\title{
ON THE DISTANCE OF THE GLOBULAR CLUSTER M4 (NGC 6121) USING RR LYRAE STARS. I. OPTICAL AND NEAR-INFRARED PERIOD-LUMINOSITY AND PERIOD-WESENHEIT RELATIONS
}

\author{
V. F. Braga ${ }^{1,2}$, M. Dall'Ora ${ }^{3}$, G. Bono ${ }^{1,2}$, P. B. Stetson ${ }^{4}$, I. Ferraro ${ }^{2}$, G. Iannicola ${ }^{2}$, M. Marengo $^{5}$, J. Neeley $^{5}$, \\ S. E. Persson ${ }^{6}$, R. Buonanno ${ }^{1,7}$, G. Coppola ${ }^{3}$, W. Freedman ${ }^{6}$, B. F. Madore ${ }^{6}$, M. Marconi ${ }^{3}$,

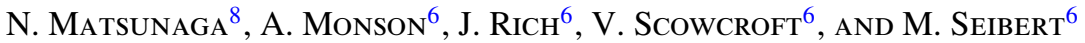 \\ ${ }^{1}$ Department of Physics, Università di Roma Tor Vergata, via della Ricerca Scientifica 1, I-00133 Roma, Italy \\ 2 INAF-Osservatorio Astronomico di Roma, via Frascati 33, I-00040 Monte Porzio Catone, Italy \\ ${ }^{3}$ INAF-Osservatorio Astronomico di Capodimonte, Salita Moiariello 16, I-80131 Napoli, Italy \\ ${ }^{4}$ NRC-Herzberg, Dominion Astrophysical Observatory, 5071 West Saanich Road, Victoria BC V9E 2E7, Canada \\ ${ }^{5}$ Department of Physics and Astronomy, Iowa State University, Ames, IA 50011, USA \\ ${ }^{6}$ Carnegie Observatories, 813 Santa Barbara Street, Pasadena, CA 91101, USA \\ ${ }^{7}$ INAF-Osservatorio Astronomico di Teramo, Via Mentore Maggini snc, Loc. Collurania, I-64100 Teramo, Italy \\ ${ }^{8}$ Kiso Observatory, Institute of Astronomy, School of Science, The University of Tokyo 10762-30, Mitake, Kiso-machi, Kiso-gun, 3 Nagano 97-0101, Japan \\ Received 2014 July 19; accepted 2014 November 24; published 2015 January 27
}

\begin{abstract}
We present new distance determinations to the nearby globular M4 (NGC 6121) based on accurate optical and near-infrared (NIR) mean magnitudes for fundamental (FU) and first overtone (FO) RR Lyrae variables (RRLs), and new empirical optical and NIR period-luminosity (PL) and period-Wesenheit (PW) relations. We have found that optical-NIR and NIR PL and PW relations are affected by smaller standard deviations than optical relations. The difference is the consequence of a steady decrease in the intrinsic spread of cluster RRL apparent magnitudes at fixed period as longer wavelengths are considered. The weighted mean visual apparent magnitude of 44 cluster RRLs is $\langle V\rangle=13.329 \pm 0.001$ (standard error of the mean) \pm 0.177 (weighted standard deviation) mag. Distances were estimated using RR Lyr itself to fix the zero-point of the empirical PL and PW relations. Using the entire sample (FU+FO) we found weighted mean true distance moduli of $11.35 \pm 0.03 \pm 0.05$ mag and $11.32 \pm 0.02 \pm$ 0.07 mag. Distances were also evaluated using predicted metallicity dependent PLZ and PWZ relations. We found weighted mean true distance moduli of $11.283 \pm 0.010 \pm 0.018 \mathrm{mag}$ (NIR PLZ) and $11.272 \pm 0.005 \pm 0.019 \mathrm{mag}$ (optical-NIR and NIR PWZ). The above weighted mean true distance moduli agree within $1 \sigma$. The same result is found from distances based on PWZ relations in which the color index is independent of the adopted magnitude $(11.272 \pm 0.004 \pm 0.013 \mathrm{mag})$. These distances agree quite well with the geometric distance provided by Kaluzny et al. based on three eclipsing binaries. The available evidence indicates that this approach can provide distances to globulars hosting RRLs with a precision better than $2 \%-3 \%$.
\end{abstract}

Key words: globular clusters: individual (M4) - stars: distances - stars: horizontal-branch stars: variables: RR Lyrae

\section{INTRODUCTION}

Globular clusters (GCs) have played a crucial role in modern astrophysics. They are fundamental laboratories not only for stellar evolution (Denissenkov \& VandenBerg 2003; Pietrinferni et al. 2006; Dotter et al. 2007; VandenBerg et al. 2012; Pietrinferni et al. 2013) and stellar dynamics (Kouwenhoven et al. 2010), but also for constraining models of Galaxy formation and evolution (Zoccali et al. 2000; MarínFranch et al. 2009; Valenti et al. 2011; Leaman et al. 2013; Johnson et al. 2013) and primordial abundances (Zoccali et al. 2003; Salaris et al. 2004; Troisi et al. 2011).

It is not surprising that the astronomical community has carried out an enormous theoretical and observational effort to properly constrain their structural parameters (Casetti-Dinescu et al. 2013; Di Cecco et al. 2013) and intrinsic properties (Gratton et al. 2004; Bono et al. 2010b; Milone et al. 2013).

Dating back to the seminal investigations by Zinn (1980) and Zinn \& West (1984) and to the more recent analysis of iron (Kraft \& Ivans 2003; Carretta et al. 2009) and $\alpha$-element abundances we have solid estimates of the metallicity scale in Galactic globulars. The same is true for the abundances of $s$ and $r$-process elements (Roederer et al. 2011; Lardo et al. 2013) and of lithium (Spite et al. 2012).
During the last few years we have also acquired a wealth of new information on the kinematic properties of halo and bulge Galactic globulars (Casetti-Dinescu et al. 2007, 2013; Vieira et al. 2007; Poleski et al. 2013). Detailed numerical simulations have also been provided for the survival rate of globulars after multiple bulge and disk crossings (Capuzzo \& Miocchi 2008).

The estimation of both absolute and relative ages of Galactic globulars has been at the crossroads of several detailed investigations (Buonanno et al. 1998; Stetson et al. 1999; Zoccali et al. 2003; Richer et al. 2004; De Angeli et al. 2005; Richer et al. 2013). The recent survey based on photometry with the Advanced Camera for Surveys on board the Hubble Space Telescope (HST) has been applied to large samples of Galactic globulars. They range from homogeneous relative ages for nine GCs by Sarajedini et al. (2007) to 64 GCs by Marín-Franch et al. (2009), to $6 \mathrm{GCs}$ by Dotter et al. (2011), and to $55 \mathrm{GCs}$ of VandenBerg et al. (2013).

The scenario outlined above indicates that we are dealing with precise and homogeneous investigations concerning age and metallicity distributions and the kinematics of Galactic globulars. However, we still lack a homogeneous distance scale for GCs. The reasons are manifold.

1. The primary distance indicators adopted to estimate absolute distances of GCs can only be applied to subsamples. 
The tip of the red giant branch (RGB) can be applied reliably only to very massive GCs, namely, $\omega$ Cen and 47 Tuc. The white dwarf cooling sequence has only been applied to nearby GCs (Zoccali et al. 2001; Richer et al. 2013). Main sequence fitting has only been applied to GCs with iron abundances bracketed by nearby dwarf stars with accurate trigonometric parallaxes (Gratton et al. 2003; Bond et al. 2013). The use of kinematic distances has also been applied only to nearby GCs (Peterson et al. 1995; Layden et al. 2005). Distances from eclipsing binaries are very precise and promising, but they have only been measured for a limited sample (Thompson et al. 2010; Kaluzny et al. 2013). The use of the predicted zero age horizontal branch (ZAHB) luminosity appears as a very promising approach (VandenBerg et al. 2013). However, uncertainties in the input physics (electron conductive opacities, Cassisi et al. 2007) and in the mass loss rate during the RGB and horizontal-branch (HB) evolutionary phases (Salaris 2012) affect the predicted luminosity of HB stellar structures.

The luminosity of the asymptotic giant branch bump has several advantages, but its application is once again limited to massive GCs (Pulone 1992; Salaris 2013). The RGB bump is also an interesting distance indicator, but predicted luminosities are at odds with observed luminosities and we still lack an accurate empirical calibration (Ferraro et al. 1999).

2. The Leavitt relation of type II Cepheids and MIRAS has also been applied to a limited number of GCs (Feast et al. 2000; Matsunaga et al. 2009). The Leavitt relation is a period-magnitude relation, but we will refer to it as a period-luminosity (PL) relation to point out the difference with the period-Wesenheit (PW) relation. The $M_{V}$ versus iron relation for RR Lyraes (RRLs) has been applied to several GCs, but their distances are affected by evolutionary effects and by a possible nonlinearity of the relation (Caputo et al. 2000). The SX Phoenicis stars have also been used to estimate the distances of a few GCs (Gilliland et al. 1998; Kaluzny \& Thompson 2009; McNamara 2011; Cohen \& Sarajedini 2012), but the physical mechanisms driving their formation and identification of their pulsation mode are still controversial (Fiorentino et al. 2013, and references therein).

3. Several of the above methods are affected by uncertainties in the cluster reddening. This problem becomes even more severe for GCs affected by differential reddening. We still lack a reddening scale based on a single diagnostic that can be used for both halo and bulge GCs.

4. Photometry and spectroscopy of cluster stars located in the innermost regions is often a difficult observational problem due to crowding. Recent empirical evidence indicates that cluster RRLs located in the central cluster regions might be contaminated by neighboring stars (Majaess et al. 2012b, 2012a). It is worth mentioning that the central density of M4 $\left(\log \rho_{V}=3.64 L_{\odot} \mathrm{pc}^{-3}\right)$ is one to two orders of magnitude smaller than GCs with high central densities $\left(\log \rho_{V}=4.6-5.6 L_{\odot} \mathrm{pc}^{-3}\right.$; Harris 1996) Additionally, the half-light radius of M4 is among the largest, at 4.33 arcmin (Harris 1996). For these reasons M4 is not nearly as severely affected by crowding problems as the bulk of Galactic globulars.

The theoretical and empirical scenario concerning absolute and relative distances to Galactic globulars (Bono et al. 2008b) is far from being satisfactory. Precise distances based on geometrical methods are limited to only a few nearby clusters. Moreover, the different standard candles are still affected by systematics that need to be constrained by independent and precise diagnostics.

In this investigation we provide a new estimate of the true distance modulus of M4 from new optical (UBVRI) and nearinfrared (NIR, $J H K$ ) photometry for RRLs in the cluster (Stetson et al. 2014). For this purpose we use optical and NIR period-luminosity-metallicity (PLZ) and period-Wesenheitmetallicity (PWZ) relations; the latter provide distances that are corrected for reddening, assuming that the reddening law is known.

The structure of the paper is as follows. In Section 2 we discuss recent distance determinations to M4, while in Section 3 we present the optical and NIR data sets used in this investigation. Then Section 4 deals with the observed optical and NIR PL relations; moreover, here we also compare to similar results available in the literature. Empirical optical, optical-NIR, and NIR PWZ relations are discussed in Section 5. In Section 6 we present new theoretical optical and NIR PLZ and PWZ relations. The true distance moduli based on the current optical and NIR photometry are discussed in Section 7. Finally, in Section 8 we summarize the results of this investigation and briefly outline the anticipated future development of the project.

\section{DISTANCE EVALUATIONS TO THE GC M4}

The distance to M4 has been estimated using several primary distance indicators, since it is the closest GC. Peterson et al. (1995) obtained a geometric distance by comparing the radial-velocity and proper-motion dispersions, finding a true distance modulus of $11.18 \pm 0.18 \mathrm{mag}$. The M4 distance was also estimated by Liu \& Janes (1990b), who applied the infrared surface-brightness technique-a variant of the Baade-Wesselink method-to four cluster RRLs (V2, V15, V32, V33); they found a true distance modulus of $11.19 \pm 0.01$ mag. Note that the stated error is only the standard error of the mean distance for the four RRLs, and does not take account of possible systematic uncertainties such as the $p$-factor that has been adopted, i.e., the parameter that transforms the observed radial velocity into a pulsation velocity (Nardetto et al. 2013). The current uncertainties in the $p$-factor imply systematic uncertainties in individual RRLs distances of the order of $10 \%$ (Marconi et al. 2005).

A similar distance to M4 was also obtained by Longmore et al. (1990) in their seminal investigation of the $K$-band PL relation for cluster RRLs. Applying a new calibration of the $K$-band PL relation to NIR photometry of 26 RRLs they found a true distance modulus of $11.28 \pm 0.06 \mathrm{mag}$ for an assumed $E(B-V)$ of 0.37 mag. A similar approach was also adopted by Bono et al. (2003), but they employed a $K$-band PLZ relation based on nonlinear pulsation models. They used the four RRLs with accurate $K$-band light curves and individual reddening estimates (Liu \& Janes 1990b) and, assuming an iron content of $[\mathrm{Fe} / \mathrm{H}]=$ -1.30 (see their Table 6), they found a true distance modulus of $11.37 \pm 0.08 \mathrm{mag}$.

The distance to M4 has also been estimated by Hendricks et al. (2012) (henceforth H12) from the HB luminosity level, and they found a true distance modulus of $11.28 \pm 0.06$ (random error) and a mean reddening $E(B-V)=0.37 \pm$ $0.01 \mathrm{mag}$. Note that $\mathrm{H} 12$ also considered uncertainties in the extinction parameter- $R_{V}$-adopted in the empirical reddening law (Cardelli et al. 1989) to constrain the selective absorption coefficients (see their Table 5). They concluded that a value 
Table 1

True Distance Moduli and Reddenings for M4 Available in the Literature

\begin{tabular}{lcccc}
\hline \hline $\begin{array}{l}\mu^{\mathrm{a}} \\
(\mathrm{mag})\end{array}$ & $\begin{array}{c}R_{V}{ }^{\mathrm{b}} \\
(\mathrm{mag})\end{array}$ & $\begin{array}{c}E(B-V)^{\mathrm{c}} \\
(\mathrm{mag})\end{array}$ & Ref. $^{\mathrm{d}}$ & Notes $^{\mathrm{e}}$ \\
\hline $11.28 \pm 0.06$ & $3.62 \pm 0.07$ & $0.37 \pm 0.01$ & $\mathrm{H} 12$ & $(1)$ \\
$11.18 \pm 0.18$ & $\ldots$ & $\ldots$ & $\mathrm{P} 95$ & $(2)$ \\
$11.19 \pm 0.01$ & 3.8 & $0.34 \pm 0.03$ & $\mathrm{LJ}$ & $(3)$ \\
$11.22 \pm 0.11$ & 4 & $0.37 \pm 0.01$ & $\mathrm{DL}$ & $(4)$ \\
$11.28 \pm 0.06$ & 4 & 0.37 & $\mathrm{~L} 90$ & $(5)$ \\
11.48 & 3.8 & 0.32 & $\mathrm{~B} 09$ & $(6)$ \\
$11.18 \pm 0.18$ & 3.8 & $0.35 \pm 0.01$ & $\mathrm{H} 04$ & $(7)$ \\
$11.30 \pm 0.05$ & $3.62 \pm 0.07$ & $0.399 \pm 0.010$ & $\mathrm{~K} 13$ & $(8)$ \\
$11.37 \pm 0.08$ & $\cdots$ & $\cdots$ & $\mathrm{B} 03$ & $(9)$ \\
\hline
\end{tabular}

Notes.

a True distance modulus and its error when estimated by the authors.

$\mathrm{b}$ The ratio between absolute and selective extinction.

c Mean reddening.

${ }^{d}$ References: H12, Hendricks et al. (2012); P95: Peterson et al. (1995); LJ: Liu \& Janes (1990b); DL: Dixon \& Longmore (1993); L90: Longmore et al. (1990); B09: Bedin et al. (2009); H04: Hansen et al. (2004); K13: Kaluzny et al. (2013); B03: Bono et al. (2003).

e (1) H12 derived a new reddening law for M4 by using both optical and NIR photometry. The true distance modulus was estimated using the zero age horizontal branch (ZAHB). (2) Astrometric distance based on proper motions and radial velocities. This distance modulus is independent of reddening uncertainties. (3) Baade-Wesselink distance based on optical near-infrared (NIR) photometry of four RR Lyrae (V2, V15, V32, V33). The individual reddening values are listed in their Table 5. They also assume an iron abundance of $[\mathrm{Fe} / \mathrm{H}]=-1.3 \pm$ 0.2 . (4) The distance is based on a new estimate of the reddening to M4, on a new metallicity $([\mathrm{Fe} / \mathrm{H}]=-1.10 \pm 0.25)$ and on distance estimates available in the literature. (5) The distance is based on the $K$-band period-luminosity (PLK) relation of RR Lyrae stars. The error on the distance is the error on the zero-point of the PLK relation of M4. The mean reddening and the mean metallicity $([\mathrm{Fe} / \mathrm{H}]=-1.28)$ are from Buonanno et al. (1989). (6) The distance is based on the fit between HB stars and the ZAHB. They provide an apparent distance modulus in $(\mathrm{m}-\mathrm{M})_{F 606 \mathrm{~W}}$ together with the extinction in the $V$ band $\left(A_{V}=1.2\right)$. They also assumed $[\mathrm{Fe} / \mathrm{H}]=-1.07 \pm 0.01([\alpha / \mathrm{Fe}]=0.39 \pm 0.05)$ by Marino et al. (2008). (7) The distance is based on different distance estimates available in the literature and in particular on the main sequence fitting provided by Richer et al. (1997). (8) The distance is based on three eclipsing binaries. The dust-type parameter was adopted by H12, the individual reddenings are listed in their Table 6. (9) Distance based on the four RR Lyrae observed by Liu $\&$ Janes (1990b) and using a theoretical $K$-band period-luminosity-metallicity relation provided by Bono et al. (see their Table 6).

of $R_{V} \sim 3.6$ was preferable to the canonical value of $\sim 3.1$, presumably related to the $\rho$ Oph star-forming cloud lying in front of the cluster.

Main-sequence fitting to field subdwarfs was adopted by Richer et al. (1997) and by Hansen et al. (2004) to estimate the distance; they found a true distance modulus $11.18 \pm 0.18 \mathrm{mag}$ for an assumed reddening of $E(B-V)=0.35 \pm 0.01 \mathrm{mag}$ and a ratio of total to selective extinction $R_{V}=3.8$. More recently, Kaluzny et al. (2013) used three detached eclipsing double-lined binary members of M4 and the reddening law found by H12 (see Table 1) to provide a true distance modulus of $11.30 \pm 0.05 \mathrm{mag}$.

The distance determinations discussed in this section suggest that estimates of the absolute distance to the closest GC range from $1.72 \pm 0.14 \mathrm{kpc}$ (Peterson et al. 1995) to $\sim 1.98 \mathrm{kpc}$ (Bedin et al. 2009). They agree within $1 \sigma$, but the full size of the confidence interval is of the order of $15 \%$ (see distance determinations listed in Table 1).

\section{OPTICAL AND NEAR-INFRARED DATA SETS}

The reader interested in a detailed discussion of the different optical and NIR data sets and the approach adopted to perform the photometry is referred to (Stetson et al. 2014). The optical photometry was transformed into the Johnson ( $U B V)$, Kron/ Cousins (RI) photometric system (Stetson 2000, 2005). The NIR photometry was transformed into the Two Micron Sky Survey (2MASS) $J H K_{s}$ photometric system (Skrutskie et al. 2006).

In the following we neglect the available $U$-band photometry because of the relatively poor time sampling and the limited accuracy of individual measurements. The optical light curves are characterized by good time sampling and the number of measurements ranges from 900 to 1100 in the $B$ band, from 1400 to 1500 in the $V$ band, from 1580 to 1800 in the $R$ band, and from 10 to 60 in the $I$ band. The NIR light curves have more limited coverage and the number of measurements ranges from five to 55 in the $J$ band, from one to nine in the $H$ band, and from two to 40 in the $K$ band.

The mean optical and NIR magnitudes were evaluated as intensity means and then transformed into magnitude. The phasing of the light curves was performed with the new period estimates provided by Stetson et al. (2014). The mean magnitudes in the bands with good time sampling $(B V R J)$ were estimated from a fit with a spline under tension. The individual mean magnitudes were estimated by equally sampling the analytical fit. The mean magnitudes in the $I$ and $K$ bands were estimated using the light curve templates provided by $\mathrm{Di}$ Criscienzo et al. (2011) and Jones et al. (1996). To apply the templates we adopted the epochs of maxima and the optical amplitudes provided by Stetson et al. (2014). For two variables not covered by our optical photometry, we adopted epochs of maxima and amplitudes available in the literature. The reader interested in a more detailed discussion concerning the amplitude ratio between optical and NIR magnitudes is referred to Stetson et al. (2014).

The number of candidate cluster RRLs is currently 44 (31 fundamental $=$ "FU" pulsators, and 13 first overtone = "FO" pulsators) and their periods range from 0.2275 to 0.6270 days plus a single long-period FU RRLs with $P=0.8555$ days. The presence of such a long-period variable is consistent with the tail in the period distribution of $\omega$ Cen RRLs found by Marconi et al. (2011). On the other hand, Andrievsky et al. (2010) suggested that at least some field long-period RRLs, such as KP Cyg, appear to be metal-rich plus $\mathrm{C}$ - and $\mathrm{N}$-enhanced. Therefore, they suggested that these objects could be short-period BL Her stars, defining a new class of variable stars, instead of long-period RRL. The extended spectroscopic analysis of both evolved and main sequence stars performed by Malavolta et al. (2014) does not support the presence of a spread in metal abundance in M4. However, we still lack detailed information concerning CNO abundances among cluster HB stars.

The current empirical and theoretical evidence indicates that the RRLs do obey a PL relation. The key feature is that the slope is negative for wavelengths longer than the $V$ band, while it is positive at shorter wavelengths. In the $V$ band the slope attains a negligible value. This is the main reason why the $M_{V}^{R R}$ versus $[\mathrm{Fe} / \mathrm{H}]$ relation was so popular in the last century to estimate the distance of both cluster and field RRLs. Plain physics arguments suggest that the occurrence of well defined NIR PL relations for RRLs is due to a significant change in the NIR bolometric corrections when moving from the blue (short periods) to the red (long period) edge of the instability strip. This change is vanishing in the $V$ band and becomes of the order of $1.5 \mathrm{mag}$ in 
the $K$ band (see Figure 1 in Bono 2003). This working hypothesis was also supported in an independent theoretical investigation by Catelan et al. (2004). Further evidence for a lack of PL relations in $B$ and $V$ bands was brought forward by Benkho et al. (2006) using accurate photometry for more than 220 RRLs in M3 (see their Figure 8). The NIR PL relations of RRLs became quite popular as distance indicators soon after their empirical determination (Longmore et al. 1986, 1990; Jones et al. 1988; Liu \& Janes 1990a, 1990b; Carney et al. 1992). A new spin was then provided by theoretical pulsation and evolutionary predictions (Bono et al. 2001, 2003; Catelan et al. 2004; Cassisi et al. 2004). The observational scenario was also significantly improved by the use of the new NIR arrays (Dall'Ora et al. 2004; Del Principe et al. 2005), the 2MASS photometry (Sollima et al. 2006), and the first accurate trigonometric parallax for RR Lyr itself (van Altena et al. 1995; Perryman et al. 1997; Benedict et al. 2002). The trigonometric parallax of RR Lyr was also adopted to fix the zero-point of both the theoretical (Bono et al. 2002) and empirical $K$-band PL relations (Sollima et al. 2006, 2008).

Pros and cons of optical-NIR PL relations and of the $M_{V}^{R R}$ versus $[\mathrm{Fe} / \mathrm{H}]$ relations have been widely discussed in the literature. In passing, we mention that the optical, NIR, and mid-infrared (MIR) PL relations for RRLs appear to be linear over the entire period range (Bono et al. 2001, 2003; Catelan et al. 2004; Madore et al. 2013). The above diagnostics are prone to uncertainties in the reddening corrections and in the adopted reddening law. Obviously, the problem becomes less and less severe when moving from the optical to the NIR and MIR bands. The impact when compared with the $V$ band is 10 times smaller in the $K$ band and more than 20 times smaller in the $3.6 \mu \mathrm{m}$ band.

The key advantage in dealing with the RRLs in M4 is that quantities necessary for calculating the distance moduli, such as the mean reddening $(E(B-V)=0.37 \pm 0.01 \mathrm{mag})$, the ratio of total to selective absorption $\left(R_{V}=3.62 \pm 0.07 \mathrm{mag}\right)$ and the overall reddening law have been recently provided by H12 (see their Table 5).

On the basis of the current mean magnitudes we estimated optical $(R I)$ and NIR $(J H K)$ PL relations. We decided to provide independent PL relations for FO and FU pulsators. The reasons are threefold. (1) Empirical and theoretical evidence indicates that the width in temperature of the region in which FO variables are pulsationally stable is roughly a factor of two narrower than the region in which FU variables are pulsationally stable. This means that the standard deviations of FO PL relations are, at fixed photometric precision, intrinsically smaller than for FU PL relations. (2) Smaller standard deviations imply more accurate relative and absolute distances. (3) The light curves of FO pulsators are more nearly sinusoidal and show pulsation amplitudes that are on average from two to three times smaller than FU variables. This means that a more limited number of measurements can provide accurate mean magnitudes. The main drawback is that FO variables are typically $\sim 0.5 \mathrm{mag}$ fainter than FU variables in the longer-wavelength bands.

However, the number of FO variables in M4 is modest and to improve the precision of the empirical PL relations we also derived PL relations from the entire sample of FU and FO variables. The global PL relations were evaluated by fundamentalizing FO periods according to the relation $\log P_{F}=\log P_{\mathrm{FO}}+0.127$. This approach to fundamentalizing the period of FO variables relies on the assumption that the period ratio of double-mode RRLs attains a constant value of the order of 0.746 . The above assumption was supported by former theoretical and empirical evidence (Iben \& Huchra 1971; Rood 1973; Cox et al. 1983). However, recent findings indicate that the double-mode field and cluster RRLs do cover a significant range in period ratios $(\sim 0.735-0.750$; G. Coppola et al. 2015, in preparation). The same outcome results from nonlinear pulsation predictions (M. Marconi et al. 2015, in preparation). We plan to address this issue in a future paper. The zero-points, the slopes, and their errors and standard deviations are listed in Table 2. The data given in this table support the above contention that the standard deviations of FO PL relations are smaller than FU PL relations, and these are in turn smaller than FU+FO PL relations (see vertical error bars). There is also evidence that the zero-points and the slopes of both optical and NIR PL relations agree within one $\sigma$. This finding might be affected by the limited sample of FO variables in M4. We plan to address this issue in a future paper in which we will deal with larger samples of cluster variables (V. F. Braga et al. 2015, in preparation).

The data plotted in Figure 1 show that the intrinsic dispersion of the PL relations decreases steadily when moving from the optical to the NIR bands. The standard deviation in the $R$ band is a factor of two larger than in the $K$ band. The reasons for the difference were mentioned above. In passing, we also note that the slope of the $K$-band PL relation is a factor of three steeper than the $R$-band PL relation. This means that the use of a PL instead of a period-luminosity-color (PLC) relation-i.e., neglecting the width in temperature of the instability strip-becomes more valid when moving from the optical to the NIR (Bono et al. 2010a; Coppola et al. 2011) and MIR (Madore et al. 2013) bands.

Finally, we mention that candidate Blazhko RRLs (black crosses) seem to follow, within the errors, PL relations similar to singly periodic FU variables.

\section{OBSERVED OPTICAL, OPTICAL-NIR, AND NIR PERIOD-WESENHEIT RELATIONS}

The key advantages in using PW relations in estimating individual distances are several. (1) They are independent of reddening uncertainties and of differential reddening provided the form of the reddening law is known (Van den Bergh 1975; Madore 1982). (2) They mimic a PLC relation so they can provide, in contrast with the PL relation, individual distances (Bono et al. 2008a; Inno et al. 2013). They are also affected by two drawbacks. (1) They require accurate mean magnitudes in a minimum of two photometric bands, and this limitation becomes more severe in dealing with optical-NIR photometry; (2) they rely on the assumption that the reddening law is known. It is well understood that this working hypothesis is not always valid in low-latitude Galactic regions. This limitation does not apply to M4, however, since H12 derived a reddening law specific to M4 by considering both optical and NIR photometry. Our current Wesenheit magnitudes have been estimated using the absorption coefficient ratios listed in Table 5 of H12.

Figure 2 displays the six optical PW relations for FU and FO pulsators. These show several distinctive features.

1. The intrinsic dispersion is, at fixed period, smaller than in the optical PL relations. This difference is expected because we already mentioned that the PW relations mimic a PLC relation. Moreover, the dispersion of the individual data points decreases in Wesenheit magnitudes based on 


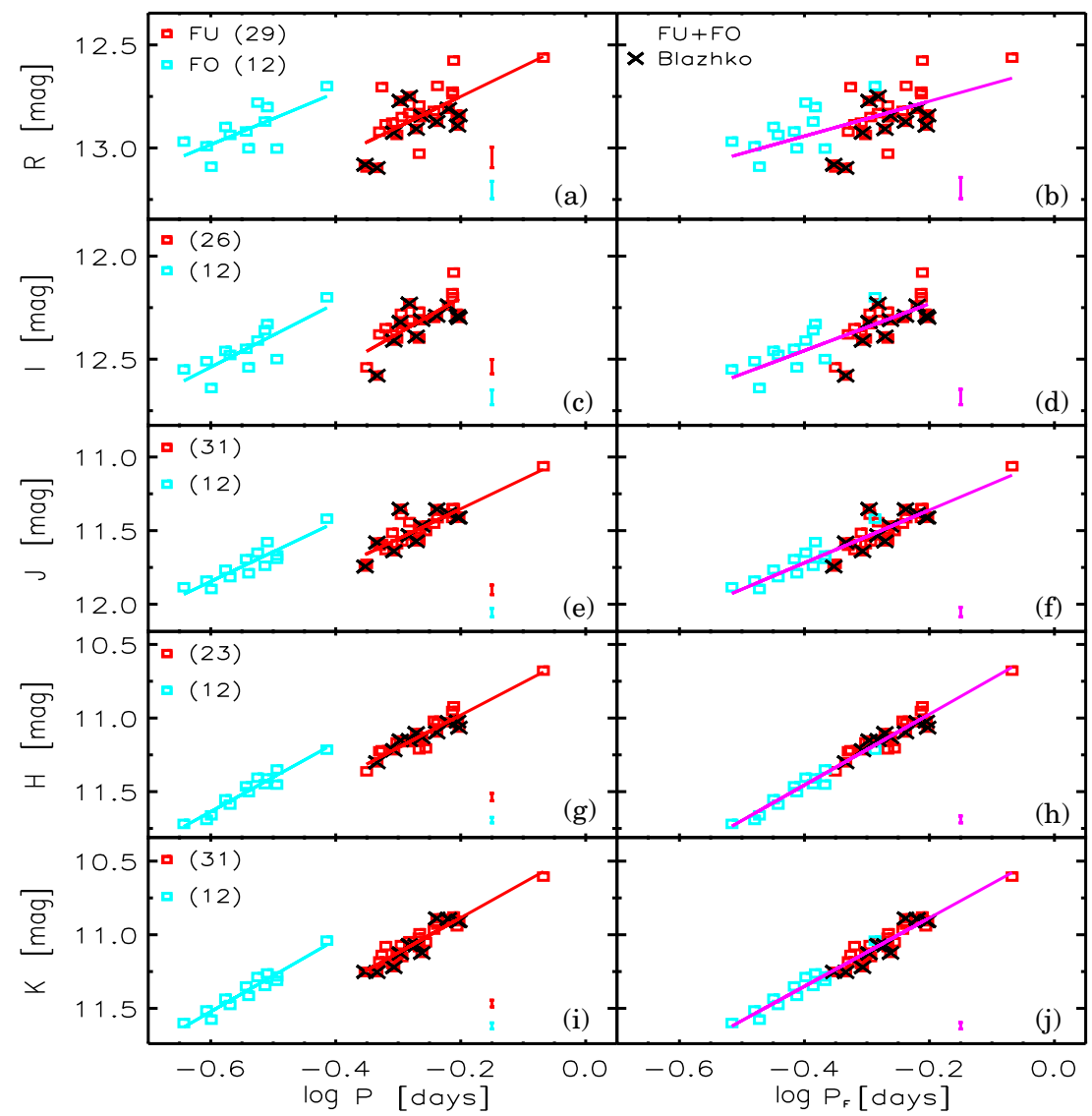

Figure 1. From top to bottom observed optical and NIR period-luminosity (PL) relations for RR Lyrae in M4. Panel (a): $R$-band period-luminosity (PL) relation Fundamental (FU) and first overtone (FO) pulsators are marked with red diamonds and cyan squares, respectively. The black crosses display candidate Blazkho RR Lyrae. The cyan and the red lines display the linear fits, while the vertical bars show the standard deviations, $\sigma$, of the fits. The number of variables adopted in the fits are also labeled. Panel (b): same as panel (a), but for FU and FO RR Lyrae. The periods of FO variables were fundamentalized using the relation: $\log P_{\mathrm{FU}}=\log P_{\mathrm{FO}}+0.127$. Panels (c) and (d): same as panels (a) and (b), but for the $I$-band PL relation. Panels (e) and (f): same as panels (a) and (b), but for the $J$-band PL relation. Panels (g) and (h): same as panels (a) and (b), but for the $H$-band PL relation. Panels (i) and (j): same as panels (a) and (b), but for the $K$-band PL relation.

Table 2

Observed Optical and NIR Period-Luminosity Relations for RR Lyrae in M4

\begin{tabular}{lccccccccc}
\hline \hline $\mathrm{Mag}^{\mathrm{a}}$ & $\begin{array}{c}a^{\mathrm{b}} \\
(\mathrm{mag})\end{array}$ & $\begin{array}{c}b^{\mathrm{b}} \\
(\mathrm{mag})\end{array}$ & $\begin{array}{c}\sigma^{\mathrm{b}} \\
(\mathrm{mag})\end{array}$ & $\begin{array}{c}a^{\mathrm{c}} \\
(\mathrm{mag})\end{array}$ & $\begin{array}{c}b^{\mathrm{c}} \\
(\mathrm{mag})\end{array}$ & $\begin{array}{c}\sigma^{\mathrm{c}} \\
(\mathrm{mag})\end{array}$ & $\begin{array}{c}a^{\mathrm{d}} \\
(\mathrm{mag})\end{array}$ & $\begin{array}{c}b^{\mathrm{d}} \\
(\mathrm{mag})\end{array}$ & $\begin{array}{c}\sigma^{\mathrm{d}} \\
(\mathrm{mag})\end{array}$ \\
\hline & & $\mathrm{FO}$ & & & $\mathrm{FU}$ & & & $\mathrm{FU}+\mathrm{FO}$ & \\
& 12.228 & -1.260 & 0.084 & 12.456 & -1.472 & 0.099 & 12.604 & -0.847 & 0.103 \\
& \pm 0.230 & \pm 0.420 & & \pm 0.085 & \pm 0.313 & & \pm 0.057 & \pm 0.177 & \\
& 11.609 & -1.549 & 0.072 & 11.858 & -1.724 & 0.070 & 12.004 & -1.137 & 0.075 \\
& \pm 0.195 & \pm 0.356 & & \pm 0.085 & \pm 0.311 & & \pm 0.047 & \pm 0.144 & \\
& 10.634 & -2.020 & 0.056 & 10.946 & -2.030 & 0.065 & 11.002 & -1.793 & 0.064 \\
& \pm 0.148 & \pm 0.273 & & \pm 0.056 & \pm 0.204 & & \pm 0.035 & \pm 0.109 & \\
$H$ & 10.232 & -2.340 & 0.037 & 10.537 & -2.215 & 0.050 & 10.492 & -2.408 & 0.046 \\
& \pm 0.097 & \pm 0.179 & & \pm 0.047 & \pm 0.176 & & \pm 0.027 & \pm 0.082 & \\
$K$ & 10.058 & -2.440 & 0.041 & 10.410 & -2.372 & 0.045 & 10.420 & -2.326 & 0.043 \\
& \pm 0.108 & \pm 0.198 & & \pm 0.039 & \pm 0.142 & & \pm 0.024 & \pm 0.074 & \\
\hline
\end{tabular}

Notes.

${ }^{a}$ PL relations of the form: $M_{X}=\mathrm{a}+\mathrm{b} \times \log P$.

${ }^{\mathrm{b}}$ Zero-point (a), slope (b) and standard deviation ( $\sigma$ ) for first overtone (FO) pulsators. The errors on the zero-point and on the slope are listed in the second row.

${ }^{\mathrm{c}}$ Zero-point (a), slope (b) and standard deviation ( $\sigma$ ) for fundamental (FU) pulsators. The errors on the zero-point and on the slope are listed in the second row.

${ }^{\mathrm{d}}$ Zero-point (a), slope (b) and standard deviation $(\sigma)$ for for the entire sample (FU + FO) of RR Lyrae. The periods of FO variables were fundamentalized by adopting the following relation: $\log P_{F}=\log P_{\mathrm{FO}}+0.127$. The errors on the zero-point and on the slope are listed in the second row. 


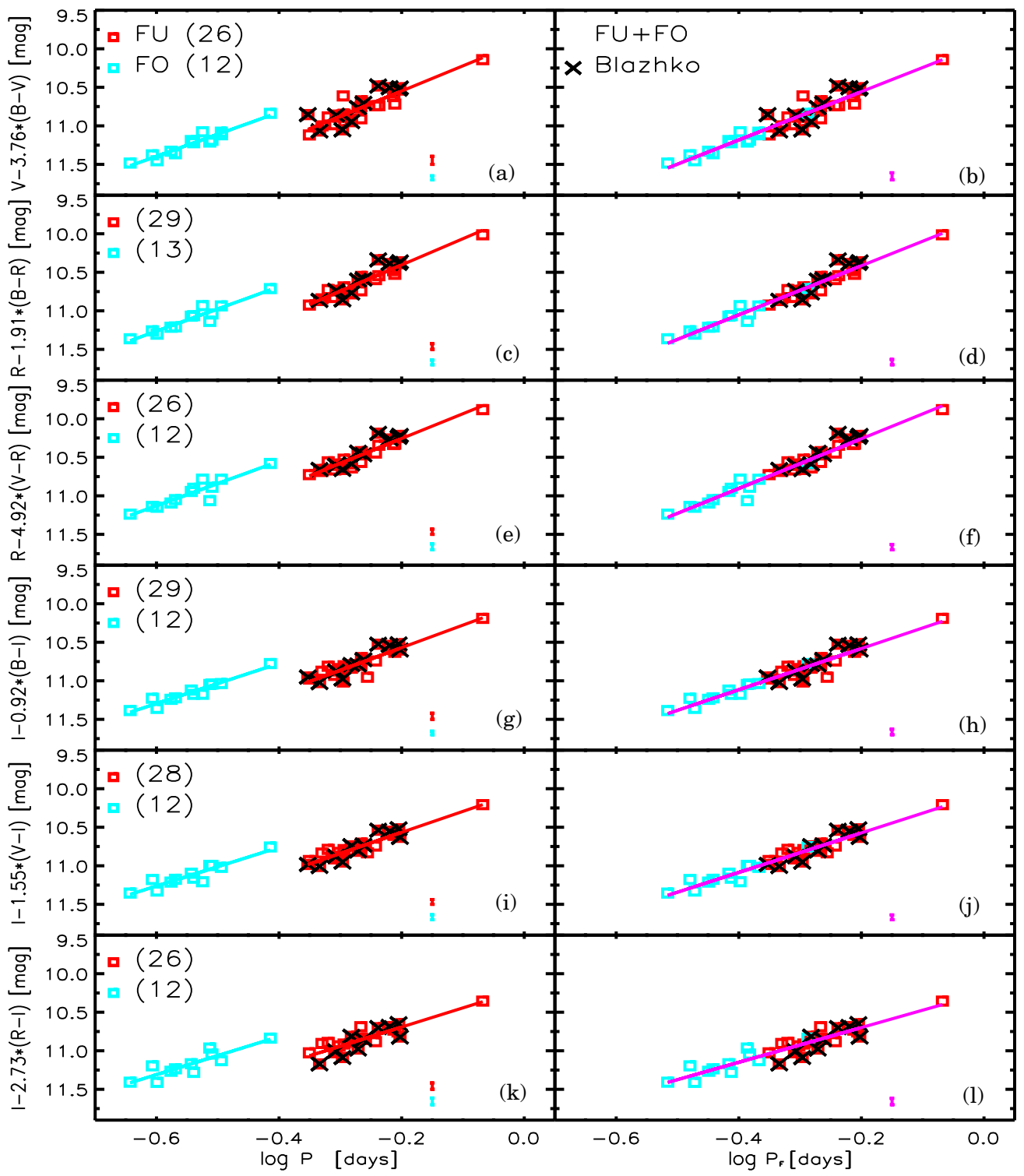

Figure 2. From top to bottom observed optical period-Wesenheit (PW) relations for RR Lyrae in M4. Symbols and lines are the same as in Figure 1. Panel (a): PW(V, $B-V$ ) relation for FU and FO pulsators. Panel (b): same as panel (a), but for the entire sample of RR Lyrae. The periods of FO variables were fundamentalized using the relation: $\log P_{F U}=\log P_{\mathrm{FO}}+0.127$. Panels (c) and (d): same as panels (a) and (b), but for the $\mathrm{PW}(R, B-R$ ) relation. Panels (e) and (f): same as panels (a) and (b), but for the $\mathrm{PW}(R, V-R)$ relation. Panels (g) and (h): same as panels (a) and (b), but for the PW(I,B-I) relation. Panels (i) and (j): same as panels (a) and (b), but for the PW(I, $V-I)$ relation. Panels (k) and (l): same as panels (a) and (b), but for the PW(I, $R-I)$ relation.

photometric bands that have a large difference in central wavelengths. This is caused by the fact that the coefficients of the color term in the Wesenheit magnitudes may attain values smaller than unity. This means that the $W(I, B-I)$ index is less prone to uncertainties affecting the mean color than the $W(I, R-I)$ index ( 0.92 versus 2.73$)$. Moreover, the increased difference in central wavelength also means an increased sensitivity to the mean effective temperature of the variable.

2. The global PW relations including both FU and FO pulsators are characterized by smaller intrinsic dispersions and by smaller errors in both the zero-point and the slope (see Table 3) when compared with FU and FO individual PW relations). This difference is caused by the increase in sample size and, in particular, by the larger range in period covered by FU plus FO variables. However, the global PW relations might be affected by the assumption that the difference between FU and FO PW relations is only a difference in the zero-point. The slopes of the global PW relations do attain values that are intermediate between the slopes of FU and FO PW relations. However, the current sample does not allow us to constrain this effect quantitatively.

3. The slopes of the PW relations listed in Columns 3, 6, and 9 of Table 3 become steeper, as expected, when moving from Wesenheit magnitudes based on $V$ and $R$ magnitudes to those based on I magnitudes (Catelan et al. 2004; Bono et al. 2010a; Madore et al. 2013).

4. The candidate Blazhko variables display, within the errors, similar slopes to those of the canonical FU variables. This further supports the inference that the mean magnitudes and colors of the Blazhko variables are minimally affected by the secondary modulations, if the primary modulation is properly covered in the two adopted bands. 
Table 3

Observed Optical Period-Wesenheit Relations for RR Lyrae in M4

\begin{tabular}{|c|c|c|c|c|c|c|c|c|c|c|}
\hline $\mathrm{PW}^{\mathrm{a}}$ & $x^{\mathrm{b}}$ & $\begin{array}{c}a^{\mathrm{c}} \\
(\mathrm{mag})\end{array}$ & $\begin{array}{c}b^{\mathrm{c}} \\
(\mathrm{mag})\end{array}$ & $\begin{array}{c}\sigma^{\mathrm{c}} \\
(\mathrm{mag})\end{array}$ & $\begin{array}{c}a^{\mathrm{d}} \\
(\mathrm{mag})\end{array}$ & $\begin{array}{c}b^{\mathrm{d}} \\
(\mathrm{mag})\end{array}$ & $\begin{array}{c}\sigma^{\mathrm{d}} \\
(\mathrm{mag})\end{array}$ & $\begin{array}{c}a^{\mathrm{e}} \\
(\mathrm{mag})\end{array}$ & $\begin{array}{c}b^{\mathrm{e}} \\
(\mathrm{mag})\end{array}$ & $\begin{array}{c}\sigma^{\mathrm{e}} \\
(\mathrm{mag})\end{array}$ \\
\hline & & FO & & & $\mathrm{FU}$ & & & $\mathrm{FU}+\mathrm{FO}$ & & \\
\hline \multirow[t]{2}{*}{$V, B-V$} & 3.76 & 9.68 & -2.86 & 0.05 & 9.89 & -3.27 & 0.10 & 9.93 & -3.13 & 0.09 \\
\hline & & \pm 0.14 & \pm 0.25 & & \pm 0.09 & \pm 0.33 & & \pm 0.05 & \pm 0.16 & \\
\hline \multirow[t]{2}{*}{$R, B-R$} & 1.91 & 9.53 & -2.89 & 0.06 & 9.72 & -3.40 & 0.07 & 9.78 & -3.18 & 0.07 \\
\hline & & \pm 0.17 & \pm 0.30 & & \pm 0.07 & \pm 0.25 & & \pm 0.04 & \pm 0.12 & \\
\hline \multirow[t]{2}{*}{$R, V-R$} & 4.92 & 9.39 & -2.89 & 0.08 & 9.61 & -3.22 & 0.07 & 9.61 & -3.23 & 0.07 \\
\hline & & \pm 0.21 & \pm 0.38 & & \pm 0.06 & \pm 0.23 & & \pm 0.04 & \pm 0.12 & \\
\hline \multirow[t]{2}{*}{$I, B-I$} & 0.92 & 9.73 & -2.60 & 0.05 & 9.98 & -2.93 & 0.08 & 10.05 & -2.67 & 0.07 \\
\hline & & \pm 0.13 & \pm 0.24 & & \pm 0.07 & \pm 0.26 & & \pm 0.04 & \pm 0.12 & \\
\hline \multirow[t]{2}{*}{$I, V-I$} & 1.55 & 9.76 & -2.51 & 0.06 & 10.02 & -2.72 & 0.06 & 10.06 & -2.56 & 0.06 \\
\hline & & \pm 0.18 & \pm 0.32 & & \pm 0.05 & \pm 0.20 & & \pm 0.03 & \pm 0.11 & \\
\hline \multirow[t]{2}{*}{$I, R-I$} & 2.73 & 9.83 & -2.47 & 0.09 & 10.19 & -2.48 & 0.08 & 10.25 & -2.25 & 0.08 \\
\hline & & \pm 0.24 & \pm 0.44 & & \pm 0.07 & \pm 0.28 & & \pm 0.05 & \pm 0.15 & \\
\hline
\end{tabular}

Notes.

${ }^{\mathrm{a}} \mathrm{PW}$ relations of the form: $W\left(M_{1}, M_{2}-M_{3}\right)=\mathrm{a}+\mathrm{b} \times \log P$.

${ }^{b}$ Color coefficient in Wesenheit magnitude: $x_{W\left(M_{1}, M_{2}-M_{3}\right)}=1 /\left(A_{M_{2}} / A_{M_{1}}-A_{M_{3}} / A_{M_{2}}\right)$.

${ }^{c}$ Zero-point (a), slope (b) and standard deviation $(\sigma)$ for FO variables. The errors on the zero-point and on the slope are listed in the second row.

d Zero-point (a), slope (b) and standard deviation ( $\sigma$ ) for FU variables. The errors on the zero-point and on the slope are listed in the second row.

e Zero-point (a), slope (b) and standard deviation $(\sigma)$ for the entire sample (FU+FO) of RR Lyrae. The periods of FO variables were fundamentalized by adopting the following relation: $\log P_{F}=\log P_{\mathrm{FO}}+0.127$. The errors on the zero-point and on the slope are listed in the second row.

Figures 3 and 4 show the observed optical-NIR and NIR PW relations. The coefficients of the PW relations and their dispersions are listed in Table 4. The trend is similar to the optical PW relations: the dispersion decreases with increasing difference in the central wavelengths of the adopted color. The above empirical evidence is the consequence of two independent mechanisms. (1) The increased difference in central wavelength causes a substantial change in the color coefficient, and indeed it ranges from $4.92 \mathrm{PW}(R, V-R)$ to $0.11 \mathrm{PW}(K, B-K)$. In particular, the optical-NIR PW relations have color coefficients that are systematically smaller than unity. This means that they are less affected by uncertainties in the mean colors. Note that the PW $(I$, $B-I)$ relation is also characterized by a color coefficient that is smaller than unity, but the errors in the coefficients of this relation are on average larger when compared with optical-NIR PW relations. (2) The increased difference in central wavelength implies a stronger sensitivity to the mean effective temperature, and in turn a more uniform distribution of RRLs across the instability strip and along the PW relation.

The dispersion of FU pulsators in the NIR PW relation (panels (i) and (j) of Figure 4) is larger than for the FO PW relations. Although the latter are fainter they also have smaller luminosity amplitudes, and their mean NIR colors are on average more accurate. This is the reason why we did not include the $\mathrm{PW}(H$, $J-H)$ and the $\mathrm{PW}(K, H-K)$ relations, the $J-H$ and the $H-K$ colors being less accurate.

To fully exploit the power of the current optical and NIR RRLs mean magnitudes for the distance of M4 we also adopted the three-band PW relations. These are PW relations in which the pass-bands adopted in the color index differ from the pass-band adopted for the magnitude. Among all the possible combinations we only selected PW relations in which the coefficient of the color index is smaller than unity. Figure 5 shows the nine empirical PW relations we selected, while Table 4 gives the coefficients, their uncertainties, and the standard deviations.
The data listed in Table 4 indicate that both the uncertainties in the coefficients and the standard deviations of the optical-NIR, three-band PW relations (Riess et al. 2011) are up to a factor of two smaller than in the optical-NIR, two-band ones.

\section{THEORETICAL PERIOD-LUMINOSITY-METALLICITY AND PERIOD-WESENHEIT-METALLICITY RELATIONS}

To estimate the distance to M4 we adopted theoretical PLZ and PWZ relations. The reasons are twofold. (1) The five field RRLs for which accurate trigonometric parallaxes are available do not yet have accurate optical and NIR mean magnitudes (Benedict et al. 2011). The only exception is RR Lyr itself for which accurate $B V$ (Szeidl 1997) and NIR Sollima et al. (2008) mean magnitudes are available in the literature. (2) Absolute distances based on predicted PL relations for RRLs do agree quite well with similar cluster distances based on solid distance indicators (Cassisi et al. 2004; Del Principe et al. 2006; Sollima et al. 2006; Bono et al. 2011; Coppola et al. 2011).

To provide a detailed theoretical framework for both PLZ and PWZ relations we adopted the large set of nonlinear, convective pulsation models recently computed by M. Marconi et al. (2015, in preparation). Models were computed for both FU and FO pulsators and cover a broad range in metal abundance $(-2.62 \leqslant$ $[\mathrm{Fe} / \mathrm{H}] \leqslant-0.29)$. The stellar masses and the luminosity were fixed by using evolutionary prescriptions for HB models provided (Pietrinferni et al. 2004, 2006). The reader interested in more details concerning the physical and numerical assumptions adopted to construct the pulsation models is referred to Bono \& Stellingwerf 1994, M. Marconi et al. 2015, in preparation and references therein. To take account of the metallicity dependence M. Marconi et al. (2015, in preparation) performed a linear fit of FU and FO pulsators including a metallicity term. In this context it is worth mentioning that the coefficients of the metallicity term 


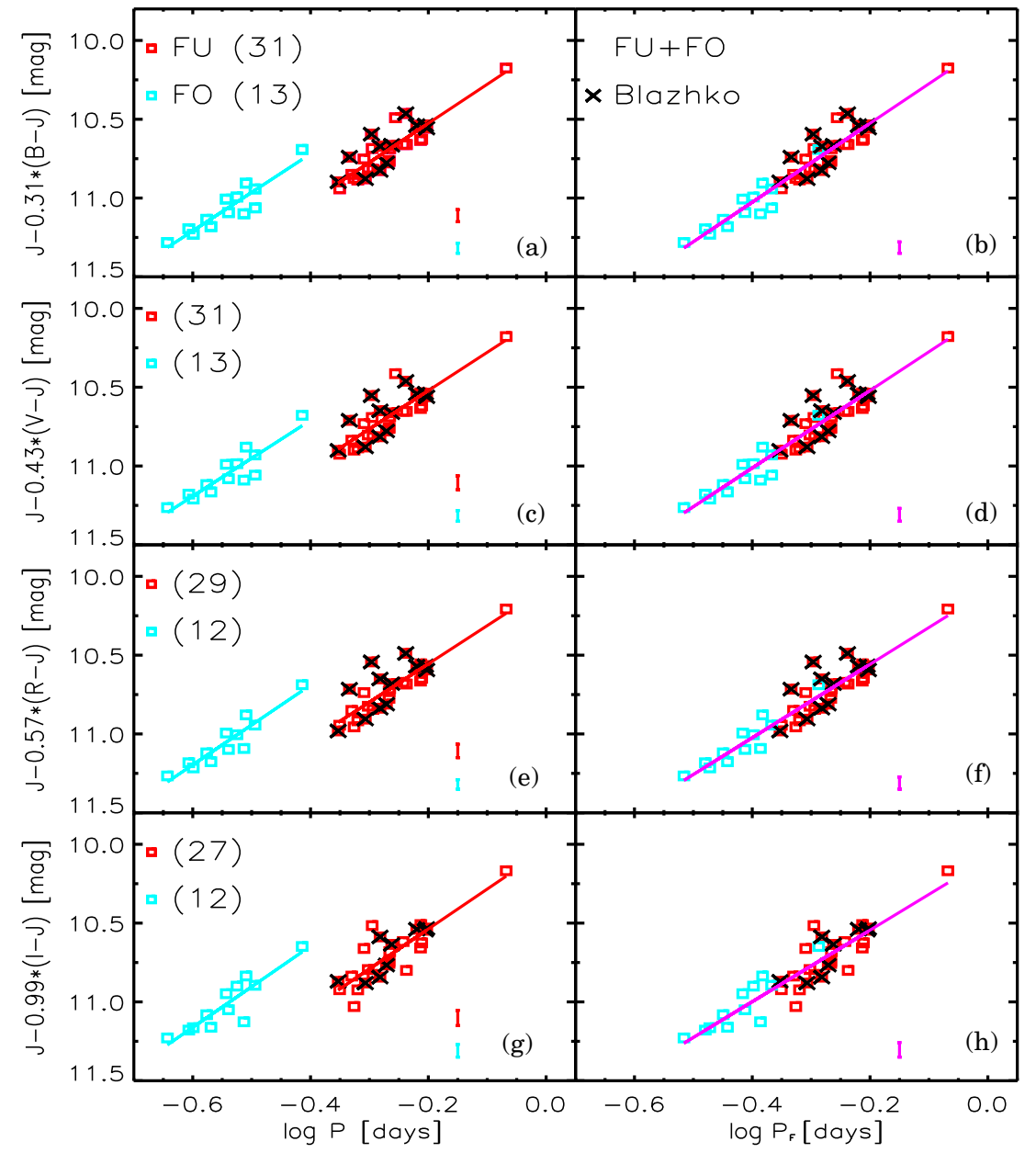

Figure 3. From top to bottom observed optical-NIR PW relations for RR Lyrae in M4. Symbols and lines are the same as in Figure 1. Panel (a): PW(J, $B-J)$ relation for FU and FO pulsators. Panel (b): same as panel (a), but for the entire sample of RR Lyrae. The periods of FO variables were fundamentalized using the relation: $\log P_{\mathrm{FU}}=\log P_{\mathrm{FO}}+0.127$. Panels (c) and (d): same as panels (a) and (b), but for the PW(J, $\left.V-J\right)$ relation. Panels (e) and (f): same as panels (a) and (b), but for the $\mathrm{PW}(J, R-J)$ relation. Panels $(\mathrm{g})$ and $(\mathrm{h})$ : same as panels (a) and (b), but for the $\mathrm{PW}(J, I-J)$ relation.

in the PLZ relations attain very similar values in both the optical and NIR bands.

The theoretical predictions were transformed into the observational plane by adopting bolometric corrections and color-temperature relations provided by (Castelli et al. 1997a, 1997b). In passing we note that above transformations are based on static, LTE atmosphere models. The use of static atmosphere models in dealing with the atmospheres of variable stars has already been addressed by (Bono et al. 1994). The nonLTE effects have impact on individual lines of individual elements, but they minimally affect broad-band colors of RRLs (see Figure 11 in Kudritzki 1979).

The HB evolutionary models we adopted to compute the mass-luminosity relation do not take account of any possible rotation. The empirical scenario concerning the rotational velocity of RRLs is far from being settled. Dating back almost $20 \mathrm{yr}$ ago, in a seminal investigation using roughly two dozen field RRLs Peterson et al. (1996) found an upper limit to the equatorial rotational velocity of $V_{\text {rot }} \sin i<10 \mathrm{~km} \mathrm{~s}^{-1}$. A more tight constraint was recently provided by Preston \& Chadid (2013) using thousands of high-resolution spectra for three dozen field RRLs. They found an upper limit $V_{\text {rot }} \sin i<6 \mathrm{~km} \mathrm{~s}^{-1}$. The above findings suggest that rotation plays a minor, if any, role in shaping the atmospheric properties of RRLs.
The PWZ relations were computed following the same approach adopted for the PLZ relations. Figures 6 and 7 display optical and NIR PW relations. The coefficients, their errors and the standard deviations are listed in Table 5.

The theoretical framework concerning the PWZ relations shows several interesting features. The optical Wesenheit magnitudes- $W(V, B-V), W(R, B-R)$ - display a peculiar trend with metallicity. An increase in metallicity from $Z=0.0001$ to $Z=0.001$ makes $W$, at fixed period, fainter while for still more metal-rich structures it becomes brighter. This is the reason why the coefficients of the metallicity term attain, for the quoted PWZ relations, vanishing values and why their standard deviations are larger. The ranking with the metallicity becomes linear for the $\operatorname{PWZ}(R, B-R)$ relation and increases for the PWZ relations including the $I$ band. The coefficient of the metallicity term attains, once again, very similar values in both the optical-NIR and NIR PWZ relations.

Figure 8 shows the nine predicted optical-NIR, three-band PW relations, while Table 5 gives the coefficients, their uncertainties and the standard deviations. The current predictions indicate that both the uncertainties in the coefficients and the standard deviations of the three-band PWZ relations attain similar values when compared with two-band PWZ relations. This evidence suggests that the improvement in the empirical 


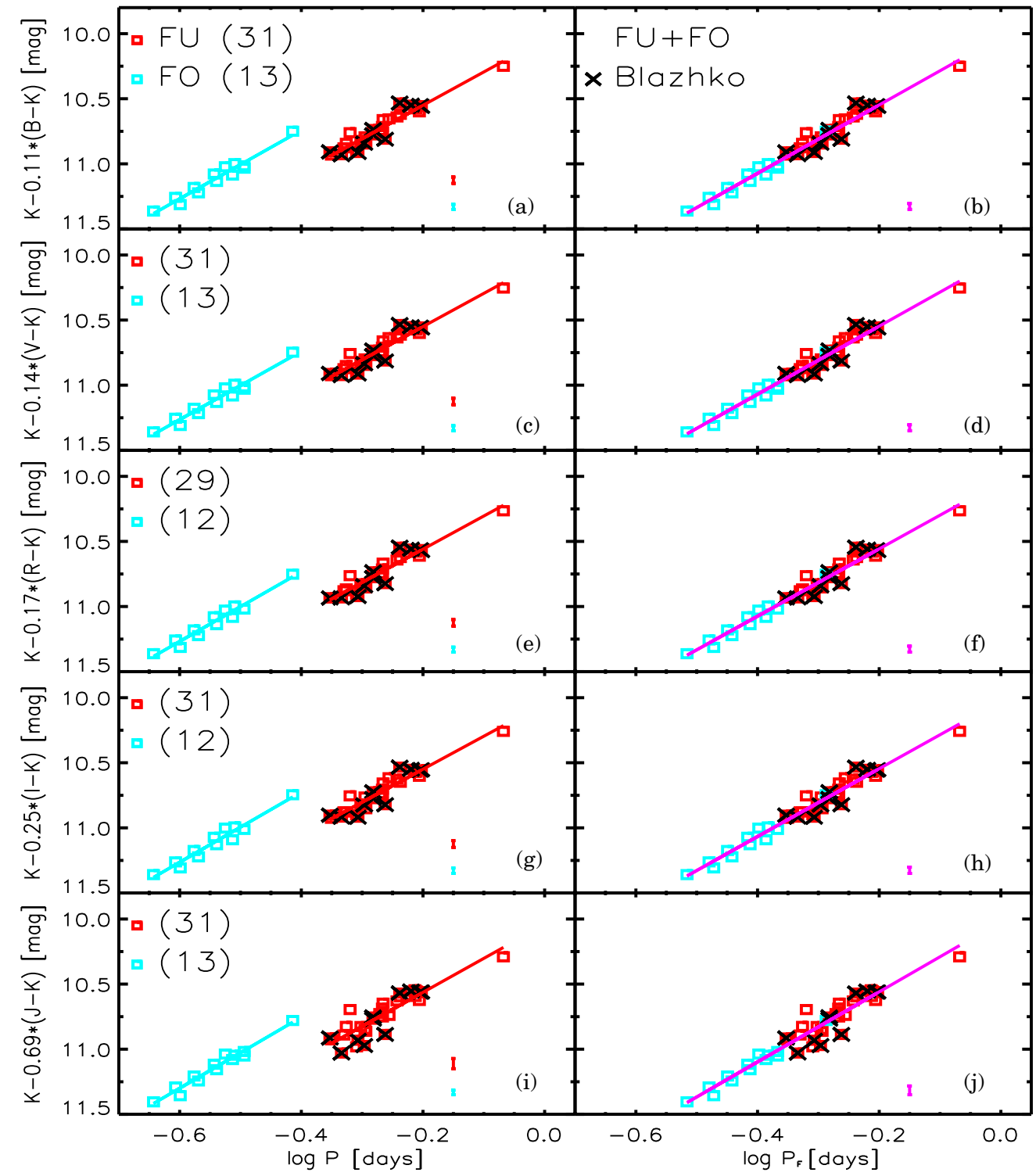

Figure 4. From top to bottom observed optical-NIR and NIR PW relations for RR Lyrae in M4. Symbols and lines are the same as in Figure 1. Panel (a): PW( $K, B-K$ ) relation for FU and FO pulsators. Panel (b): same as panel (a), but for the entire sample of RR Lyrae. The periods of FO variables were fundamentalized using the relation: $\log P_{\mathrm{FU}}=\log P_{\mathrm{FO}}+0.127$. Panels (c) and (d): same as panels (a) and (b), but for the PW(K, $\left.V-K\right)$ relation. Panels (e) and (f): same as panels (a) and (b), but for the $\mathrm{PW}(K, R-K)$ relation. Panels (g) and (h): same as panels (a) and (b), but for the $\mathrm{PW}(K, I-K)$ relation. Panels (i) and (j): same as panels (a) and (b), but for the $\mathrm{PW}(K, J-K)$ relation.

three-band PWZ relations might be a consequence of the fact that the optical mean colors adopted in the three-band PWZ relations are more precise than the optical-NIR mean colors adopted in the two-band ones.

To further constrain the theoretical framework adopted to estimate the distance to M4, we performed a detailed comparison between the slope of NIR PL relations available in the literature and the slope of the current PLZ relation. Data plotted in the bottom panel of Figure 9 indicate good agreement between the predicted $K$-band slope (dashed line), the observed slope for M4 and similar estimates for Galactic globulars. The agreement appears quite good, within the errors, over the entire metallicity range. The same applies for the $J$ band (top panel), but theory and observations agree within $\sim 1 \sigma$. We cannot reach a firm conclusion concerning the $H$ band, since only two empirical estimates are available and they attain intermediate values.

\section{DISTANCE DETERMINATIONS TO M4 BASED ON EMPIRICAL AND PREDICTED PERIOD-LUMINOSITY AND PERIOD-WESENHEIT RELATIONS}

\subsection{Cluster Distances based on the Absolute Distance of RR Lyr Itself}

Thanks to the use of the Fine Guidance Sensor on board the HST, Benedict et al. (2011) provided accurate estimates of the trigonometric parallaxes for five field RRL: SU Dra, XZ Cyg, RZ Cep, XZ Cyg, and RR Lyr. To fix the zero-points of the empirical relations we decided to use RR Lyr itself. The reasons are the following.

(1) The absolute parallax to RR Lyr itself is the most precise (3.77 \pm 0.13 mas) among the calibrating RRLs and an accurate estimate of the reddening toward RR Lyr is also available $(E(B-V)=0.02 \pm 0.03$; Sollima et al. 2008). (2) Accurate optical (BV; Szeidl 1997) and NIR (JHK; Sollima et al. 2008) 


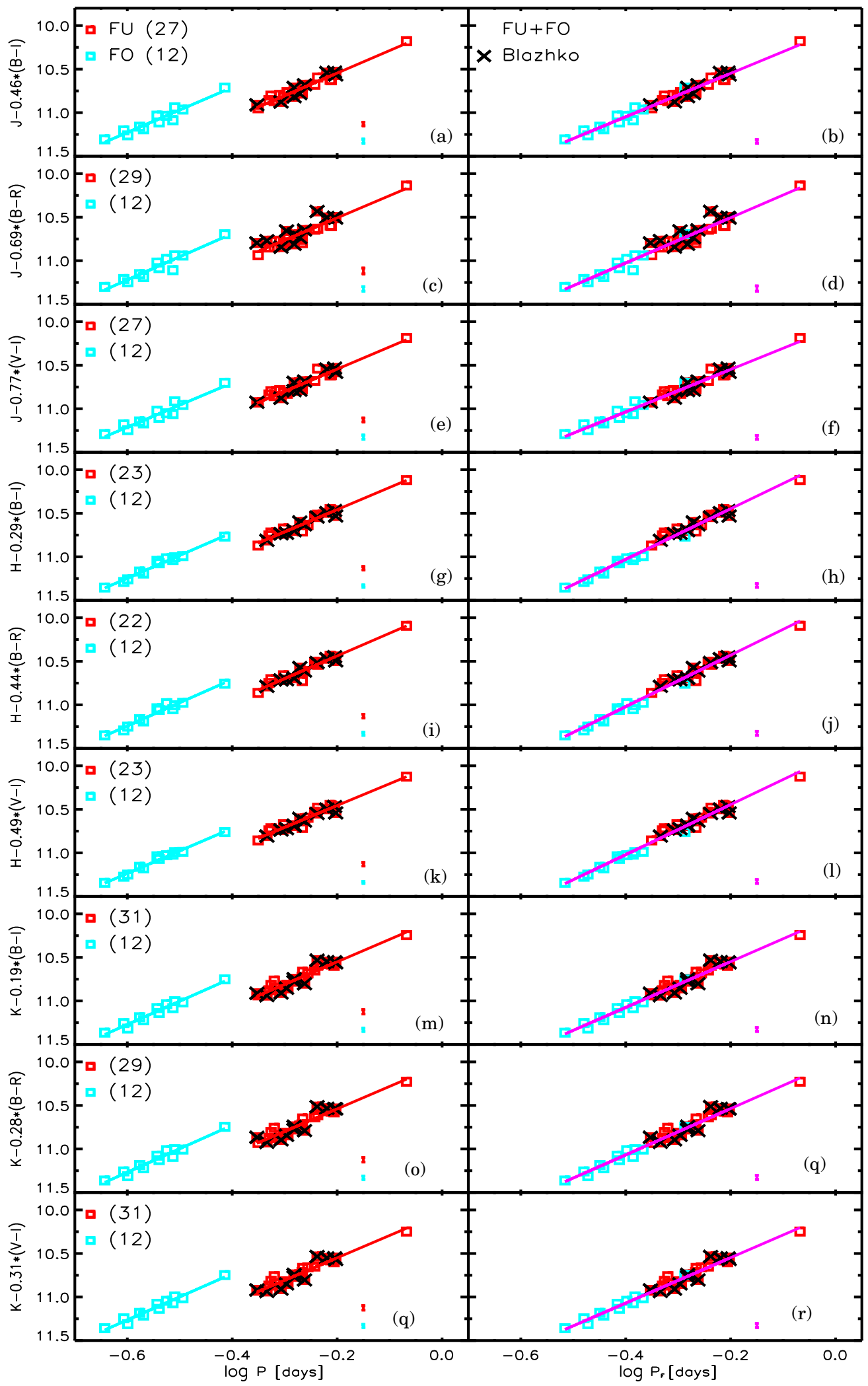

Figure 5. From top to bottom observed optical-NIR three-band PW relations for RR Lyrae in M4. Symbols and lines are the same as in Figure 1. (a): PW( $J$, $B-I)$ relation for FU and FO pulsators. (b): same as panel (a), but for the entire sample of RR Lyrae. The periods of FO variables were fundamentalized using the relation: $\log P_{\mathrm{FU}}=\log P_{\mathrm{FO}}+0.127$. (c) and (d): same as panels (a) and (b), but for the $\mathrm{PW}(J, B-R)$ relation. (e) and (f): same as panels (a) and (b), but for the PW( $\left.J, V-I\right)$ relation. (g) and (h): same as panels (a) and (b), but for the $\mathrm{PW}(H, B-I)$ relation. (i) and (j): same as panels (a) and (b), but for the PW( $H, B-R)$ relation. (k) and (l): same as panels (a) and (b), but for the $\mathrm{PW}(H, V-I)$ relation. (m) and (n): same as panels (a) and (b), but for the $\mathrm{PW}(K, B-I)$ relation. (o) and (p): same as panels (a) and (b), but for the $\mathrm{PW}(K, B-R)$ relation. (q) and (r): same as panels (a) and (b), but for the $\mathrm{PW}(K, V-I)$ relation. 
Table 4

Observed Optical-NIR Period-Wesenheit Relations for RR Lyrae in M4

\begin{tabular}{|c|c|c|c|c|c|c|c|c|c|c|}
\hline $\mathrm{PW}^{\mathrm{a}}$ & $x^{\mathrm{b}}$ & $\begin{array}{c}a^{\mathrm{c}} \\
(\mathrm{mag})\end{array}$ & $\begin{array}{c}b^{\mathrm{c}} \\
(\mathrm{mag})\end{array}$ & $\begin{array}{c}\sigma^{\mathrm{c}} \\
(\mathrm{mag})\end{array}$ & $\begin{array}{c}a^{\mathrm{d}} \\
(\mathrm{mag})\end{array}$ & $\begin{array}{c}b^{\mathrm{d}} \\
(\mathrm{mag})\end{array}$ & $\begin{array}{c}\sigma^{\mathrm{d}} \\
(\mathrm{mag})\end{array}$ & $\begin{array}{c}a^{\mathrm{e}} \\
(\mathrm{mag})\end{array}$ & $\begin{array}{c}b^{\mathrm{e}} \\
(\mathrm{mag})\end{array}$ & $\begin{array}{c}\sigma^{\mathrm{e}} \\
(\mathrm{mag})\end{array}$ \\
\hline & & $\mathrm{FO}$ & & & $\mathrm{FU}$ & & & $\mathrm{FU}+\mathrm{FO}$ & & \\
\hline$J, B-J$ & 0.31 & $\begin{array}{r}9.74 \\
\pm 0.17\end{array}$ & $\begin{array}{l}-2.44 \\
\pm 0.31\end{array}$ & 0.06 & $\begin{array}{r}10.03 \\
\pm 0.07\end{array}$ & $\begin{array}{l}-2.48 \\
\pm 0.24\end{array}$ & 0.08 & $\begin{array}{r}10.02 \\
\pm 0.04\end{array}$ & $\begin{array}{l}-2.50 \\
\pm 0.12\end{array}$ & 0.07 \\
\hline$J, V-J$ & 0.43 & $\begin{array}{r}9.75 \\
\pm 0.18\end{array}$ & $\begin{array}{l}-2.41 \\
\pm 0.33\end{array}$ & 0.07 & $\begin{array}{r}10.04 \\
\pm 0.08\end{array}$ & $\begin{array}{l}-2.43 \\
\pm 0.28\end{array}$ & 0.09 & $\begin{array}{r}10.03 \\
\pm 0.04\end{array}$ & $\begin{array}{l}-2.46 \\
\pm 0.14\end{array}$ & 0.08 \\
\hline$J, R-J$ & 0.57 & $\begin{array}{r}9.68 \\
\pm 0.16\end{array}$ & $\begin{array}{l}-2.53 \\
\pm 0.30\end{array}$ & 0.06 & $\begin{array}{r}10.07 \\
\pm 0.07\end{array}$ & $\begin{array}{l}-2.43 \\
\pm 0.27\end{array}$ & 0.08 & $\begin{array}{r}10.09 \\
\pm 0.04\end{array}$ & $\begin{array}{l}-2.33 \\
\pm 0.13\end{array}$ & 0.08 \\
\hline$J, I-J$ & 0.99 & $\begin{array}{r}9.62 \\
\pm 0.22\end{array}$ & $\begin{array}{l}-2.58 \\
\pm 0.40\end{array}$ & 0.08 & $\begin{array}{r}10.03 \\
\pm 0.08\end{array}$ & $\begin{array}{l}-2.53 \\
\pm 0.31\end{array}$ & 0.10 & $\begin{array}{r}10.09 \\
\pm 0.05\end{array}$ & $\begin{array}{l}-2.27 \\
\pm 0.16\end{array}$ & 0.09 \\
\hline$H, B-H$ & 0.18 & $\begin{array}{r}9.65 \\
\pm 0.08\end{array}$ & $\begin{array}{l}-2.64 \\
\pm 0.15\end{array}$ & 0.03 & $\begin{array}{r}9.94 \\
\pm 0.04\end{array}$ & $\begin{array}{l}-2.51 \\
\pm 0.13\end{array}$ & 0.04 & $\begin{array}{r}9.84 \\
\pm 0.03\end{array}$ & $\begin{array}{l}-2.94 \\
\pm 0.08\end{array}$ & 0.05 \\
\hline$H, V-H$ & 0.24 & $\begin{array}{r}9.65 \\
\pm 0.09\end{array}$ & $\begin{array}{l}-2.63 \\
\pm 0.16\end{array}$ & 0.03 & $\begin{array}{r}9.94 \\
\pm 0.04\end{array}$ & $\begin{array}{l}-2.48 \\
\pm 0.14\end{array}$ & 0.04 & $\begin{array}{r}9.84 \\
\pm 0.03\end{array}$ & $\begin{array}{l}-2.93 \\
\pm 0.09\end{array}$ & 0.05 \\
\hline$H, R-H$ & 0.30 & $\begin{array}{r}9.68 \\
\pm 0.09\end{array}$ & $\begin{array}{l}-2.60 \\
\pm 0.17\end{array}$ & 0.03 & $\begin{array}{r}9.96 \\
\pm 0.04\end{array}$ & $\begin{array}{l}-2.45 \\
\pm 0.15\end{array}$ & 0.04 & $\begin{array}{r}9.85 \\
\pm 0.03\end{array}$ & $\begin{array}{l}-2.91 \\
\pm 0.09\end{array}$ & 0.05 \\
\hline$H, I-H$ & 0.46 & $\begin{array}{r}9.64 \\
\pm 0.13\end{array}$ & $\begin{array}{l}-2.63 \\
\pm 0.24\end{array}$ & 0.05 & $\begin{array}{r}9.93 \\
\pm 0.05\end{array}$ & $\begin{array}{l}-2.45 \\
\pm 0.19\end{array}$ & 0.05 & $\begin{array}{r}9.80 \\
\pm 0.04\end{array}$ & $\begin{array}{l}-2.98 \\
\pm 0.11\end{array}$ & 0.06 \\
\hline$K, B-K$ & 0.11 & $\begin{array}{r}9.69 \\
\pm 0.10\end{array}$ & $\begin{array}{l}-2.63 \\
\pm 0.19\end{array}$ & 0.04 & $\begin{array}{r}10.04 \\
\pm 0.04\end{array}$ & $\begin{array}{l}-2.56 \\
\pm 0.15\end{array}$ & 0.05 & $\begin{array}{r}10.02 \\
\pm 0.02\end{array}$ & $\begin{array}{l}-2.63 \\
\pm 0.08\end{array}$ & 0.05 \\
\hline$K, V-K$ & 0.14 & $\begin{array}{r}9.69 \\
\pm 0.10\end{array}$ & $\begin{array}{l}-2.62 \\
\pm 0.19\end{array}$ & 0.04 & $\begin{array}{r}10.04 \\
\pm 0.04\end{array}$ & $\begin{array}{l}-2.55 \\
\pm 0.16\end{array}$ & 0.05 & $\begin{array}{r}10.02 \\
\pm 0.03\end{array}$ & $\begin{array}{l}-2.62 \\
\pm 0.08\end{array}$ & 0.05 \\
\hline$K, R-K$ & 0.17 & $\begin{array}{r}9.66 \\
\pm 0.10\end{array}$ & $\begin{array}{l}-2.67 \\
\pm 0.19\end{array}$ & 0.04 & $\begin{array}{r}10.05 \\
\pm 0.04\end{array}$ & $\begin{array}{l}-2.55 \\
\pm 0.16\end{array}$ & 0.05 & $\begin{array}{r}10.04 \\
\pm 0.03\end{array}$ & $\begin{array}{l}-2.59 \\
\pm 0.08\end{array}$ & 0.05 \\
\hline$K, I-K$ & 0.25 & $\begin{array}{r}9.65 \\
\pm 0.11\end{array}$ & $\begin{array}{l}-2.70 \\
\pm 0.21\end{array}$ & 0.04 & $\begin{array}{r}10.04 \\
\pm 0.04\end{array}$ & $\begin{array}{l}-2.53 \\
\pm 0.16\end{array}$ & 0.05 & $\begin{array}{r}10.02 \\
\pm 0.03\end{array}$ & $\begin{array}{l}-2.61 \\
\pm 0.08\end{array}$ & 0.05 \\
\hline$K, J-K$ & 0.69 & $\begin{array}{r}9.66 \\
\pm 0.09\end{array}$ & $\begin{array}{l}-2.73 \\
\pm 0.17\end{array}$ & 0.03 & $\begin{array}{r}10.04 \\
\pm 0.07\end{array}$ & $\begin{array}{l}-2.61 \\
\pm 0.25\end{array}$ & 0.08 & $\begin{array}{r}10.02 \\
\pm 0.04\end{array}$ & $\begin{array}{l}-2.69 \\
\pm 0.12\end{array}$ & 0.07 \\
\hline \multicolumn{11}{|c|}{ Three-bands } \\
\hline$J, B-I$ & 0.46 & $\begin{array}{r}9.67 \\
\pm 0.13\end{array}$ & $\begin{array}{l}-2.59 \\
\pm 0.23\end{array}$ & 0.05 & $\begin{array}{r}10.03 \\
\pm 0.04\end{array}$ & $\begin{array}{l}-2.59 \\
\pm 0.13\end{array}$ & 0.04 & $\begin{array}{r}10.05 \\
\pm 0.02\end{array}$ & $\begin{array}{l}-2.49 \\
\pm 0.07\end{array}$ & 0.04 \\
\hline$J, B-R$ & 0.69 & $\begin{array}{r}9.63 \\
\pm 0.14\end{array}$ & $\begin{array}{l}-2.66 \\
\pm 0.26\end{array}$ & 0.05 & $\begin{array}{r}10.00 \\
\pm 0.06\end{array}$ & $\begin{array}{l}-2.56 \\
\pm 0.21\end{array}$ & 0.07 & $\begin{array}{r}9.99 \\
\pm 0.03\end{array}$ & $\begin{array}{l}-2.60 \\
\pm 0.10\end{array}$ & 0.06 \\
\hline$J, V-I$ & 0.77 & $\begin{array}{r}9.69 \\
\pm 0.13\end{array}$ & $\begin{array}{l}-2.54 \\
\pm 0.24\end{array}$ & 0.05 & $\begin{array}{r}10.03 \\
\pm 0.04\end{array}$ & $\begin{array}{l}-2.56 \\
\pm 0.14\end{array}$ & 0.04 & $\begin{array}{r}10.06 \\
\pm 0.02\end{array}$ & $\begin{array}{l}-2.44 \\
\pm 0.08\end{array}$ & 0.04 \\
\hline$H, B-I$ & 0.29 & $\begin{array}{r}9.67 \\
\pm 0.07\end{array}$ & $\begin{array}{l}-2.62 \\
\pm 0.13\end{array}$ & 0.03 & $\begin{array}{r}9.95 \\
\pm 0.04\end{array}$ & $\begin{array}{l}-2.55 \\
\pm 0.13\end{array}$ & 0.04 & $\begin{array}{r}9.87 \\
\pm 0.02\end{array}$ & $\begin{array}{l}-2.90 \\
\pm 0.07\end{array}$ & 0.04 \\
\hline$H, B-R$ & 0.44 & $\begin{array}{r}9.64 \\
\pm 0.09\end{array}$ & $\begin{array}{l}-2.67 \\
\pm 0.17\end{array}$ & 0.03 & $\begin{array}{r}9.92 \\
\pm 0.04\end{array}$ & $\begin{array}{l}-2.61 \\
\pm 0.15\end{array}$ & 0.04 & $\begin{array}{r}9.84 \\
\pm 0.03\end{array}$ & $\begin{array}{l}-2.96 \\
\pm 0.08\end{array}$ & 0.05 \\
\hline$H, V-I$ & 0.49 & $\begin{array}{r}9.68 \\
\pm 0.06\end{array}$ & $\begin{array}{l}-2.59 \\
\pm 0.11\end{array}$ & 0.02 & $\begin{array}{r}9.96 \\
\pm 0.04\end{array}$ & $\begin{array}{l}-2.50 \\
\pm 0.14\end{array}$ & 0.04 & $\begin{array}{r}9.87 \\
\pm 0.03\end{array}$ & $\begin{array}{l}-2.87 \\
\pm 0.08\end{array}$ & 0.04 \\
\hline$K, B-I$ & 0.19 & $\begin{array}{r}9.66 \\
\pm 0.10\end{array}$ & $\begin{array}{l}-2.68 \\
\pm 0.18\end{array}$ & 0.04 & $\begin{array}{r}10.03 \\
\pm 0.04\end{array}$ & $\begin{array}{l}-2.58 \\
\pm 0.15\end{array}$ & 0.05 & $\begin{array}{r}10.02 \\
\pm 0.02\end{array}$ & $\begin{array}{l}-2.63 \\
\pm 0.08\end{array}$ & 0.04 \\
\hline$K, B-R$ & 0.28 & $\begin{array}{r}9.64 \\
\pm 0.10\end{array}$ & $\begin{array}{l}-2.71 \\
\pm 0.19\end{array}$ & 0.04 & $\begin{array}{r}10.02 \\
\pm 0.04\end{array}$ & $\begin{array}{l}-2.58 \\
\pm 0.16\end{array}$ & 0.05 & $\begin{array}{r}10.00 \\
\pm 0.03\end{array}$ & $\begin{array}{l}-2.66 \\
\pm 0.08\end{array}$ & 0.05 \\
\hline$K, V-I$ & 0.31 & $\begin{array}{r}9.67 \\
\pm 0.10\end{array}$ & $\begin{array}{l}-2.66 \\
\pm 0.18\end{array}$ & 0.04 & $\begin{array}{r}10.04 \\
\pm 0.04\end{array}$ & $\begin{array}{l}-2.57 \\
\pm 0.16\end{array}$ & 0.05 & $\begin{array}{r}10.03 \\
\pm 0.03\end{array}$ & $\begin{array}{l}-2.61 \\
\pm 0.08\end{array}$ & 0.05 \\
\hline
\end{tabular}

Notes.

${ }^{\text {a }} \mathrm{PW}$ relations of the form: $W\left(M_{1}, M_{2}-M_{3}\right)=\mathrm{a}+\mathrm{b} \times \log P . M_{3} \neq M_{1}$ only for three-band Wesenheit magnitudes.

${ }^{\mathrm{b}}$ Color coefficient in Wesenheit magnitude: $x_{W\left(M_{1}, M_{2}-M_{3}\right)}=1 /\left(A_{M_{2}} / A_{M_{1}}-A_{M_{3}} / A_{M_{2}}\right)$.

c Zero-point (a), slope (b) and standard deviation $(\sigma)$ for FO pulsators. The errors on zero-point and slope are listed in the second row.

d Zero-point (a), slope (b) and standard deviation $(\sigma)$ for FU pulsators. The errors on zero-point and slope are listed in the second row.

e Zero-point (a), slope (b) and standard deviation ( $\sigma$ ) for for the entire sample (FU+FO) of RR Lyrae. The periods of FO variables were fundamentalized by adopting the following relation: $\log P_{F}=\log P_{\mathrm{FO}}+0.127$. The errors on zero-point and slope are listed in the second row.

mean magnitudes are available in the literature (see Table 6). Note that RR Lyr together with UV Oct and XZ Cyg are affected by the Blazhko effect. This means that the typical uncertainty on its mean $B V$ magnitudes is of the order of 0.10 mag. (3) An accurate estimate of the iron abundance is also available $([\mathrm{Fe} / \mathrm{H}]=-1.41 \pm 0.13$; Kolenberg et al. 2010). To provide a homogeneous metallicity scale with RRLs in M4 we took account of the difference in the adopted solar iron abundance between Carretta et al. (2009) and Kolenberg et al. (2010) $([\mathrm{Fe} / \mathrm{H}] \sim-1.50 \pm 0.13$, see Table 6). The above iron abundance, once transformed from the Zinn \& West (1984) to the Carretta et al. (2009) metallicity scale, indicates that RR Lyr is $\sim 0.40$ dex more metal-poor than RRLs in M4 (see Table 6). 
Table 5

Theoretical Optical and NIR Period-Wesenheit-Metallicity Relations for RR Lyrae in M4

\begin{tabular}{|c|c|c|c|c|c|c|c|c|c|c|c|c|c|}
\hline$\overline{\mathrm{PWZ}^{\mathrm{a}}}$ & $x^{b}$ & $\begin{array}{c}a^{\mathrm{c}} \\
(\mathrm{mag})\end{array}$ & $\begin{array}{c}b^{\mathrm{c}} \\
(\mathrm{mag})\end{array}$ & $\begin{array}{c}c^{\mathrm{c}} \\
(\mathrm{mag})\end{array}$ & $\begin{array}{c}\sigma^{\mathrm{c}} \\
(\mathrm{mag})\end{array}$ & $\begin{array}{c}a^{\mathrm{d}} \\
(\mathrm{mag})\end{array}$ & $\begin{array}{c}b^{\mathrm{d}} \\
(\mathrm{mag})\end{array}$ & $\begin{array}{c}c^{\mathrm{d}} \\
(\mathrm{mag})\end{array}$ & $\begin{array}{c}\sigma^{\mathrm{d}} \\
(\mathrm{mag})\end{array}$ & $\begin{array}{c}a^{\mathrm{e}} \\
(\mathrm{mag})\end{array}$ & $\begin{array}{c}b^{\mathrm{e}} \\
(\mathrm{mag})\end{array}$ & $\begin{array}{c}c^{\mathrm{e}} \\
(\mathrm{mag})\end{array}$ & $\begin{array}{c}\sigma^{\mathrm{e}} \\
(\mathrm{mag})\end{array}$ \\
\hline \multicolumn{6}{|c|}{ FO } & \multicolumn{2}{|c|}{ FU } & \multicolumn{6}{|c|}{$\mathrm{FU}+\mathrm{FO}$} \\
\hline \multirow[t]{2}{*}{$\overline{V, B-V}$} & 3.76 & -1.800 & -2.858 & -0.007 & 0.049 & -1.487 & -3.031 & -0.066 & 0.106 & -1.444 & -2.848 & -0.043 & 0.102 \\
\hline & & \pm 0.055 & \pm 0.108 & \pm 0.009 & & \pm 0.022 & \pm 0.064 & \pm 0.011 & & \pm 0.019 & \pm 0.049 & \pm 0.009 & \\
\hline \multirow[t]{2}{*}{$R, B-R$} & 1.91 & -1.793 & -2.936 & 0.036 & 0.040 & -1.389 & -2.922 & 0.010 & 0.082 & -1.365 & -2.800 & 0.023 & 0.078 \\
\hline & & \pm 0.044 & \pm 0.088 & \pm 0.007 & & \pm 0.017 & \pm 0.050 & \pm 0.009 & & \pm 0.014 & \pm 0.038 & \pm 0.007 & \\
\hline \multirow[t]{2}{*}{$R, V-R$} & 4.92 & -1.787 & -3.016 & 0.080 & 0.031 & -1.288 & -2.810 & 0.088 & 0.060 & -1.283 & -2.750 & 0.090 & 0.056 \\
\hline & & \pm 0.035 & \pm 0.068 & \pm 0.006 & & \pm 0.013 & \pm 0.036 & \pm 0.006 & & \pm 0.010 & \pm 0.027 & \pm 0.005 & \\
\hline \multirow[t]{2}{*}{$I, B-I$} & 0.92 & -1.639 & -2.878 & 0.094 & 0.026 & -1.149 & -2.648 & 0.095 & 0.048 & -1.139 & -2.568 & 0.101 & 0.048 \\
\hline & & \pm 0.029 & \pm 0.057 & \pm 0.005 & & \pm 0.010 & \pm 0.029 & \pm 0.005 & & \pm 0.009 & \pm 0.023 & \pm 0.004 & \\
\hline \multirow{2}{*}{$I, V-I$} & 1.55 & -1.586 & -2.884 & 0.127 & 0.019 & -1.039 & -2.524 & 0.147 & 0.034 & -1.039 & -2.476 & 0.147 & 0.036 \\
\hline & & \pm 0.021 & \pm 0.041 & \pm 0.003 & & \pm 0.007 & \pm 0.021 & \pm 0.004 & & \pm 0.007 & \pm 0.017 & \pm 0.003 & \\
\hline \multirow[t]{2}{*}{$I, R-I$} & 2.73 & -1.494 & -2.824 & 0.148 & 0.016 & -0.924 & -2.392 & 0.175 & 0.031 & -0.927 & -2.350 & 0.174 & 0.034 \\
\hline & & \pm 0.018 & \pm 0.036 & \pm 0.003 & & \pm 0.006 & \pm 0.018 & \pm 0.003 & & \pm 0.006 & \pm 0.016 & \pm 0.003 & \\
\hline \multirow[t]{2}{*}{$J, B-J$} & 0.31 & -1.536 & -2.776 & 0.134 & 0.020 & -1.005 & -2.417 & 0.152 & 0.031 & -1.005 & -2.367 & 0.153 & 0.034 \\
\hline & & \pm 0.023 & \pm 0.044 & \pm 0.004 & & \pm 0.006 & \pm 0.019 & \pm 0.003 & & \pm 0.006 & \pm 0.016 & \pm 0.003 & \\
\hline \multirow[t]{2}{*}{$J, V-J$} & 0.43 & -1.512 & -2.769 & 0.146 & 0.019 & -0.961 & -2.362 & 0.172 & 0.030 & -0.965 & -2.323 & 0.171 & 0.033 \\
\hline & & \pm 0.021 & \pm 0.041 & \pm 0.003 & & \pm 0.006 & \pm 0.018 & \pm 0.003 & & \pm 0.006 & \pm 0.016 & \pm 0.003 & \\
\hline$J, R-J$ & 0.57 & -1.486 & -2.745 & 0.153 & 0.019 & -0.930 & -2.318 & 0.180 & 0.032 & -0.934 & -2.282 & 0.178 & 0.035 \\
\hline & & \pm 0.022 & \pm 0.043 & \pm 0.004 & & \pm 0.007 & \pm 0.019 & \pm 0.003 & & \pm 0.006 & \pm 0.017 & \pm 0.003 & \\
\hline$J, I-J$ & 0.99 & -1.484 & -2.724 & 0.154 & 0.021 & -0.932 & -2.299 & 0.181 & 0.033 & -0.936 & -2.264 & 0.180 & 0.036 \\
\hline & & \pm 0.023 & \pm 0.045 & \pm 0.004 & & \pm 0.007 & \pm 0.020 & \pm 0.004 & & \pm 0.007 & \pm 0.017 & \pm 0.003 & \\
\hline$H, B-H$ & 0.18 & -1.621 & -2.916 & 0.148 & 0.015 & -1.083 & -2.532 & 0.175 & 0.029 & -1.092 & -2.541 & 0.171 & 0.029 \\
\hline & & \pm 0.016 & \pm 0.032 & \pm 0.003 & & \pm 0.006 & \pm 0.018 & \pm 0.003 & & \pm 0.005 & \pm 0.014 & \pm 0.003 & \\
\hline$H, V-H$ & 0.24 & -1.612 & -2.919 & 0.156 & 0.013 & -1.062 & -2.508 & 0.187 & 0.027 & -1.075 & -2.526 & 0.182 & 0.028 \\
\hline & & \pm 0.015 & \pm 0.029 & \pm 0.002 & & \pm 0.006 & \pm 0.017 & \pm 0.003 & & \pm 0.005 & \pm 0.014 & \pm 0.003 & \\
\hline$H, R-H$ & 0.30 & -1.603 & -2.914 & 0.160 & 0.013 & -1.051 & -2.492 & 0.192 & 0.027 & -1.064 & -2.515 & 0.186 & 0.028 \\
\hline & & \pm 0.014 & \pm 0.028 & \pm 0.002 & & \pm 0.006 & \pm 0.016 & \pm 0.003 & & \pm 0.005 & \pm 0.013 & \pm 0.002 & \\
\hline$H, I-H$ & 0.46 & -1.616 & -2.925 & 0.161 & 0.013 & -1.067 & -2.505 & 0.195 & 0.027 & -1.081 & -2.535 & 0.188 & 0.028 \\
\hline & & \pm 0.014 & \pm 0.027 & \pm 0.002 & & \pm 0.006 & \pm 0.016 & \pm 0.003 & & \pm 0.005 & \pm 0.013 & \pm 0.002 & \\
\hline$K, B-K$ & 0.11 & -1.564 & -2.852 & 0.149 & 0.016 & -1.023 & -2.455 & 0.177 & 0.027 & -1.031 & -2.453 & 0.173 & 0.028 \\
\hline & & \pm 0.017 & \pm 0.034 & \pm 0.003 & & \pm 0.006 & \pm 0.016 & \pm 0.003 & & \pm 0.005 & \pm 0.014 & \pm 0.003 & \\
\hline$K, V-K$ & 0.14 & -1.557 & -2.851 & 0.154 & 0.015 & -1.009 & -2.438 & 0.184 & 0.027 & -1.019 & -2.441 & 0.179 & 0.028 \\
\hline & & \pm 0.016 & \pm 0.032 & \pm 0.003 & & \pm 0.006 & \pm 0.016 & \pm 0.003 & & \pm 0.005 & \pm 0.014 & \pm 0.003 & \\
\hline$K, R-K$ & 0.17 & -1.550 & -2.847 & 0.156 & 0.015 & -1.001 & -2.427 & 0.187 & 0.027 & -1.011 & -2.432 & 0.182 & 0.028 \\
\hline & & \pm 0.016 & \pm 0.032 & \pm 0.003 & & \pm 0.006 & \pm 0.016 & \pm 0.003 & & \pm 0.005 & \pm 0.014 & \pm 0.003 & \\
\hline$K, I-K$ & 0.25 & -1.554 & -2.848 & 0.156 & 0.015 & -1.006 & -2.429 & 0.188 & 0.027 & -1.017 & -2.438 & 0.183 & 0.028 \\
\hline & & \pm 0.016 & \pm 0.032 & \pm 0.003 & & \pm 0.006 & \pm 0.016 & \pm 0.003 & & \pm 0.005 & \pm 0.013 & \pm 0.002 & \\
\hline$K, J-K$ & 0.69 & -1.579 & -2.891 & 0.157 & 0.014 & -1.032 & -2.475 & 0.190 & 0.026 & -1.045 & -2.498 & 0.184 & 0.027 \\
\hline & & \pm 0.015 & \pm 0.030 & \pm 0.002 & & \pm 0.005 & \pm 0.016 & \pm 0.003 & & \pm 0.005 & \pm 0.013 & \pm 0.002 & \\
\hline$H, J-H$ & 1.72 & -1.731 & -3.099 & 0.167 & 0.018 & -1.184 & -2.683 & 0.206 & 0.037 & -1.206 & -2.770 & 0.195 & 0.038 \\
\hline & & \pm 0.020 & \pm 0.040 & \pm 0.003 & & \pm 0.008 & \pm 0.022 & \pm 0.004 & & \pm 0.007 & \pm 0.018 & \pm 0.003 & \\
\hline$K, H-K$ & 1.81 & -1.477 & -2.753 & 0.150 & 0.020 & -0.931 & -2.336 & 0.179 & 0.031 & -0.938 & -2.317 & 0.176 & 0.032 \\
\hline & & \pm 0.022 & \pm 0.043 & \pm 0.004 & & \pm 0.006 & \pm 0.019 & \pm 0.003 & & \pm 0.006 & \pm 0.016 & \pm 0.003 & \\
\hline & & & & & & Three-ba & & & & & & & \\
\hline$\overline{J, B-R}$ & 0.46 & -1.598 & -2.814 & 0.110 & 0.023 & -1.097 & -2.538 & 0.118 & 0.037 & -1.091 & -2.470 & 0.122 & 0.039 \\
\hline & & \pm 0.026 & \pm 0.051 & \pm 0.004 & & \pm 0.008 & \pm 0.023 & \pm 0.004 & & \pm 0.007 & \pm 0.019 & \pm 0.004 & \\
\hline$J, B-I$ & 0.69 & -1.561 & -2.800 & 0.124 & 0.021 & -1.040 & -2.472 & 0.138 & 0.033 & -1.037 & -2.415 & 0.140 & 0.036 \\
\hline & & \pm 0.023 & \pm 0.046 & \pm 0.004 & & \pm 0.007 & \pm 0.020 & \pm 0.003 & & \pm 0.007 & \pm 0.017 & \pm 0.003 & \\
\hline$J, V-I$ & 0.77 & -1.535 & -2.804 & 0.140 & 0.018 & -0.985 & -2.410 & 0.165 & 0.030 & -0.987 & -2.370 & 0.164 & 0.033 \\
\hline & & \pm 0.020 & \pm 0.040 & \pm 0.003 & & \pm 0.006 & \pm 0.018 & \pm 0.003 & & \pm 0.006 & \pm 0.016 & \pm 0.003 & \\
\hline$H, B-R$ & 0.29 & -1.647 & -2.919 & 0.131 & 0.018 & -1.129 & -2.591 & 0.150 & 0.035 & -1.133 & -2.580 & 0.149 & 0.033 \\
\hline & & \pm 0.020 & \pm 0.040 & \pm 0.003 & & \pm 0.007 & \pm 0.021 & \pm 0.004 & & \pm 0.006 & \pm 0.016 & \pm 0.003 & \\
\hline$H, B-I$ & 0.44 & -1.623 & -2.910 & 0.140 & 0.016 & -1.093 & -2.550 & 0.163 & 0.031 & -1.099 & -2.545 & 0.161 & 0.031 \\
\hline & & \pm 0.018 & \pm 0.036 & \pm 0.003 & & \pm 0.007 & \pm 0.019 & \pm 0.003 & & \pm 0.006 & \pm 0.015 & \pm 0.003 & \\
\hline$H, V-I$ & 0.49 & -1.607 & -2.912 & 0.150 & 0.014 & -1.058 & -2.511 & 0.180 & 0.028 & -1.068 & -2.517 & 0.175 & 0.029 \\
\hline & & \pm 0.016 & \pm 0.031 & \pm 0.003 & & \pm 0.006 & \pm 0.017 & \pm 0.003 & & \pm 0.005 & \pm 0.014 & \pm 0.003 & \\
\hline$K, B-R$ & 0.19 & -1.586 & -2.860 & 0.138 & 0.017 & -1.059 & -2.500 & 0.160 & 0.029 & -1.064 & -2.487 & 0.158 & 0.030 \\
\hline & & \pm 0.019 & \pm 0.038 & \pm 0.003 & & \pm 0.006 & \pm 0.018 & \pm 0.003 & & \pm 0.006 & \pm 0.014 & \pm 0.003 & \\
\hline$K, B-I$ & 0.28 & -1.571 & -2.854 & 0.144 & 0.016 & -1.035 & -2.474 & 0.169 & 0.028 & -1.042 & -2.464 & 0.166 & 0.029 \\
\hline & & \pm 0.018 & \pm 0.036 & \pm 0.003 & & \pm 0.006 & \pm 0.017 & \pm 0.003 & & \pm 0.005 & \pm 0.014 & \pm 0.003 & \\
\hline$K, V-I$ & 0.31 & -1.561 & -2.855 & 0.150 & 0.015 & -1.013 & -2.448 & 0.179 & 0.027 & -1.022 & -2.446 & 0.175 & 0.028 \\
\hline & & \pm 0.017 & \pm 0.033 & \pm 0.003 & & \pm 0.006 & \pm 0.016 & \pm 0.003 & & \pm 0.005 & \pm 0.014 & \pm 0.003 & \\
\hline
\end{tabular}

Notes.

a The PWZ relations of the form: $W\left(M_{1}, M_{2}-M_{3}\right)=\mathrm{a}+\mathrm{b} \times \log P+\mathrm{c} \times[\mathrm{Fe} / \mathrm{H}] . M_{3} \neq M_{1}$ only for three-band Wesenheit magnitudes.

${ }^{\mathrm{b}}$ Color coefficient in Wesenheit magnitude: $x_{W\left(M_{1}, M_{2}-M_{3}\right)}=1 /\left(A_{M_{2}} / A_{M_{1}}-A_{M_{3}} / A_{M_{2}}\right)$.

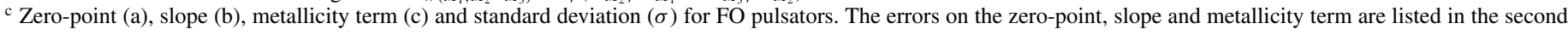
row.

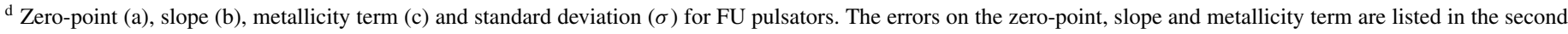
row.

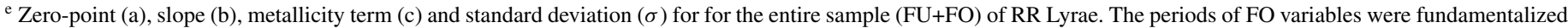
by adopting the following relation: $\log P_{F}=\log P_{\mathrm{FO}}+0.127$. The errors on the zero-point, slope and metallicity term are listed in the second row. 


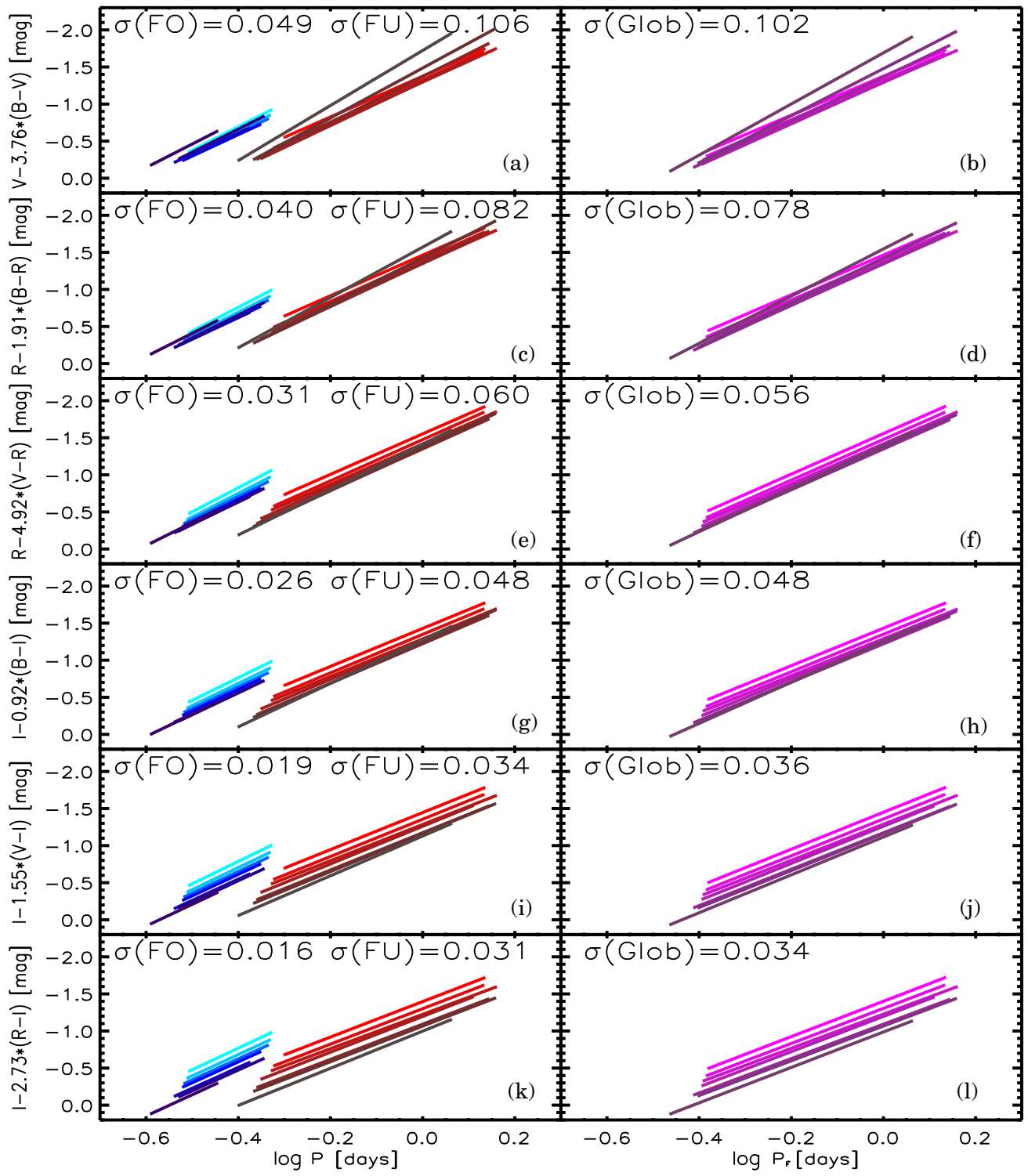

Figure 6. From top to bottom predicted optical PW relations for RR Lyrae models. The PW relations were estimated by using the reddening law for M4 provided by Hendricks et al. (2012) Panel (a): Lines of different colors display PW relations for FU and FO pulsators. The PW $(V, B-V)$ relations range in metallicity from $[\mathrm{Fe} / \mathrm{H}]=-2.62$ (brighter) to $[\mathrm{Fe} / \mathrm{H}]=-0.29$ (fainter). See Table 1 for more details concerning the adopted metallicities. Panel (b): same as panel (a), but for the entire sample of RR Lyrae models. The periods of FO models were fundamentalized using the relation: $\log P_{F U}=\log P_{\mathrm{FO}}+0.127$. Panels (c) and (d): same as panels (a) and (b), but for the predicted $\mathrm{PW}(R, B-R)$ relation. Panels (e) and (f): same as panels (a) and (b), but for the predicted PW( $R, V-R)$ relation. Panels (g) and (h): same as panels (a) and (b), but for the predicted PW $(I, B-I)$ relation. Panels (i) and (j): same as panels (a) and (b), but for the predicted PW $(I, V-I)$ relation. Panels (h) and (k): same as panels (a) and (b), but for the predicted $\mathrm{PW}(I, R-I)$ relation.

Table 6

RR Lyr Photometric and Physical Properties from Literature

\begin{tabular}{|c|c|c|c|c|c|c|c|c|}
\hline $\begin{array}{l}\text { Period }^{\mathrm{a}} \\
\text { (days) }^{\text {days }}\end{array}$ & $B^{\mathrm{b}}$ & $V^{b}$ & $\begin{array}{c}J^{\mathrm{c}} \\
(\mathrm{mag})\end{array}$ & $H^{\mathrm{c}}$ & $K^{\mathrm{c}}$ & $\begin{array}{c}\pi^{\mathrm{d}} \\
(\mathrm{mas})\end{array}$ & {$[\mathrm{Fe} / \mathrm{H}]^{\mathrm{e}}$} & $\begin{array}{c}E(B-V)^{\mathrm{f}} \\
(\mathrm{mag})\end{array}$ \\
\hline $0.5668386 \pm 0.0000016$ & $8.09 \pm 0.04$ & $7.74 \pm 0.02$ & $6.74 \pm 0.02$ & $6.60 \pm 0.03$ & $6.50 \pm 0.02$ & $3.77 \pm 0.13$ & $-1.50 \pm 0.13$ & $0.02 \pm 0.03$ \\
\hline
\end{tabular}

Notes.

${ }^{a}$ Pulsation period from Kolenberg et al. (2006).

${ }^{\text {b }}$ Intensity averaged mean magnitude estimated with a spline fit to the $B V$ photoelectric photometry provided by Szeidl (1997).

${ }^{\mathrm{c}}$ Intensity averaged mean $J H K$ magnitude (2MASS, photometric system) provided by Sollima et al. (2008).

${ }^{\mathrm{d}}$ Trigonometric parallax from Benedict et al. (2002).

${ }^{\mathrm{e}}$ Iron abundance provided by Kolenberg et al. (2010), note that to provide an homogeneous metallicity scale, we took account for the difference in the solar iron abundance adopted by Kolenberg et al. $\left(2010, \log \epsilon_{\mathrm{Fe}}=7.45\right.$, Asplund et al. (2005)) and by Carretta et al. $\left(2009, \log \epsilon_{\mathrm{Fe}}=7.54\right.$, Gratton et al. (2003)) in defining the GC metallicity scale.

${ }^{\mathrm{f}}$ Reddening according to Sollima et al. (2008). 


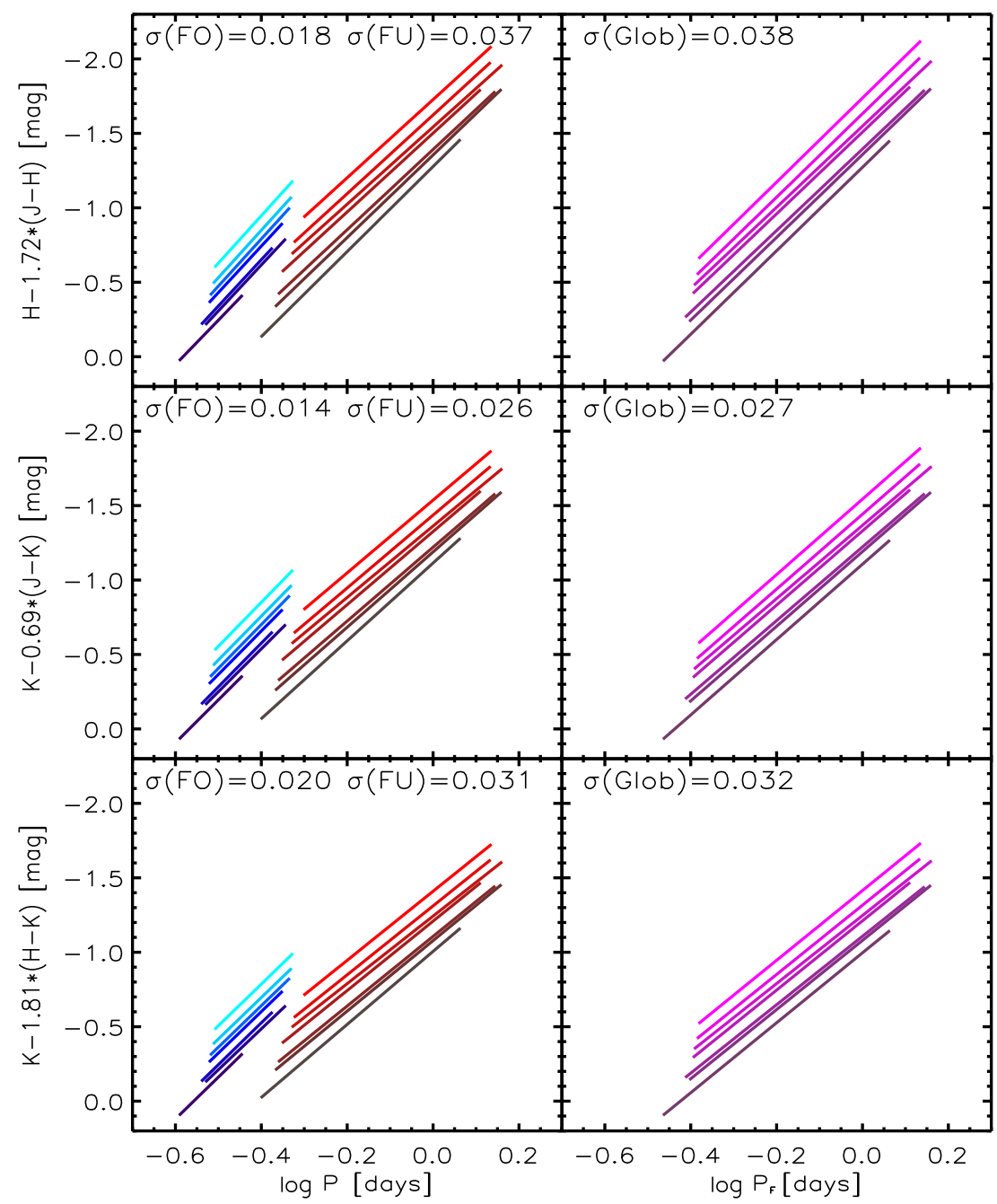

Figure 7. Same as Figure 6, but for the predicted NIR PW relations.

To provide an empirical estimate of the absolute distance to M4, we used the slopes of both PL and PW relations listed in Tables 2-4. The extinction corrections to the observed mean magnitudes have been estimated using the Cardelli et al. (1989) semi-empirical reddening law. The apparent magnitudes have also been corrected for the difference in iron abundance $(\Delta[\mathrm{Fe} / \mathrm{H}]=-0.40)$ between M4 and RR Lyr using the metallicity coefficients of the predicted PLZ and PWZ relations (see Section 5). The absolute distances based on the NIR PL relations listed in Table 7 give a weighted mean distance modulus of $11.35 \pm 0.03 \pm 0.05 \mathrm{mag}(\mathrm{FU})$ and $11.35 \pm 0.03 \pm 0.05 \mathrm{mag}$ $(\mathrm{FO}+\mathrm{FU})$. The former error is the error on the mean, while the latter is the standard deviation. The two estimates are, within the errors, identical. They also agree, within $1 \sigma$, with accurate estimates available in literature (see Table 1) and with distances based on predicted PLZ and PWZ relations (see Section 6.2). The RR Lyr zero-point was also used for the eight optical-NIR PW relations including $B V J H K$ bands. The weighted mean distance modulus is $11.31 \pm 0.02 \pm 0.07 \mathrm{mag}$ (FU) and $11.32 \pm$ $0.02 \pm 0.07 \mathrm{mag}(\mathrm{FO}+\mathrm{FU})$, respectively. The weighted mean distance modulus only based on optical-NIR and on NIR PW relations is $11.31 \pm 0.02 \pm 0.06 \mathrm{mag}(\mathrm{FU})$ and $11.32 \pm 0.02 \pm$ $0.06 \mathrm{mag}(\mathrm{FO}+\mathrm{FU})$. The above estimates agree quite well with similar estimates available in the literature and within $1 \sigma$ with distances based on predicted PWZ relations.
Table 7

True Distance Moduli Based on Observed Period-Luminosity and Period-Wesenheit Relations Calibrated with RR Lyr

\begin{tabular}{|c|c|c|c|c|}
\hline PL-PW & $\mu^{\mathrm{b}} \quad$ (mag) & $\sigma_{\mu}^{c}$ & $\mu^{\mathrm{b}} \quad$ (mag) & $\sigma_{\mu}^{\mathrm{c}}$ \\
\hline & \multicolumn{2}{|l|}{$\mathrm{FU}$} & \multicolumn{2}{|l|}{$\mathrm{FU}+\mathrm{FO}$} \\
\hline \multicolumn{5}{|c|}{ PL } \\
\hline$J$ & $11.374 \pm 0.010$ & 0.056 & $11.368 \pm 0.009$ & 0.059 \\
\hline$H$ & $11.296 \pm 0.011$ & 0.054 & $11.298 \pm 0.008$ & 0.048 \\
\hline$K$ & $11.382 \pm 0.008$ & 0.044 & $11.382 \pm 0.006$ & 0.042 \\
\hline \multicolumn{5}{|c|}{ PW } \\
\hline$V, B-V$ & $11.166 \pm 0.019$ & 0.103 & $11.159 \pm 0.014$ & 0.090 \\
\hline$J, B-J$ & $11.333 \pm 0.012$ & 0.067 & $11.329 \pm 0.009$ & 0.063 \\
\hline$J, V-J$ & $11.342 \pm 0.013$ & 0.073 & $11.337 \pm 0.010$ & 0.068 \\
\hline$H, B-H$ & $11.245 \pm 0.009$ & 0.043 & $11.251 \pm 0.008$ & 0.047 \\
\hline$H, V-H$ & $11.246 \pm 0.010$ & 0.047 & $11.252 \pm 0.008$ & 0.050 \\
\hline$K, B-K$ & $11.362 \pm 0.008$ & 0.047 & $11.364 \pm 0.007$ & 0.044 \\
\hline$K, V-K$ & $11.364 \pm 0.008$ & 0.047 & $11.367 \pm 0.007$ & 0.044 \\
\hline$K, J-K$ & $11.378 \pm 0.013$ & 0.073 & $11.383 \pm 0.009$ & 0.063 \\
\hline
\end{tabular}

Notes.

a Adopted PL or PW relation.

${ }^{\mathrm{b}}$ Mean distance modulus and its error based on $\mathrm{FU}$ and $\mathrm{FU}+\mathrm{FO}$ variables.

${ }^{\mathrm{c}}$ Standard deviation of the distance modulus based on $\mathrm{FU}$ and $\mathrm{FU}+\mathrm{FO}$ variables. 


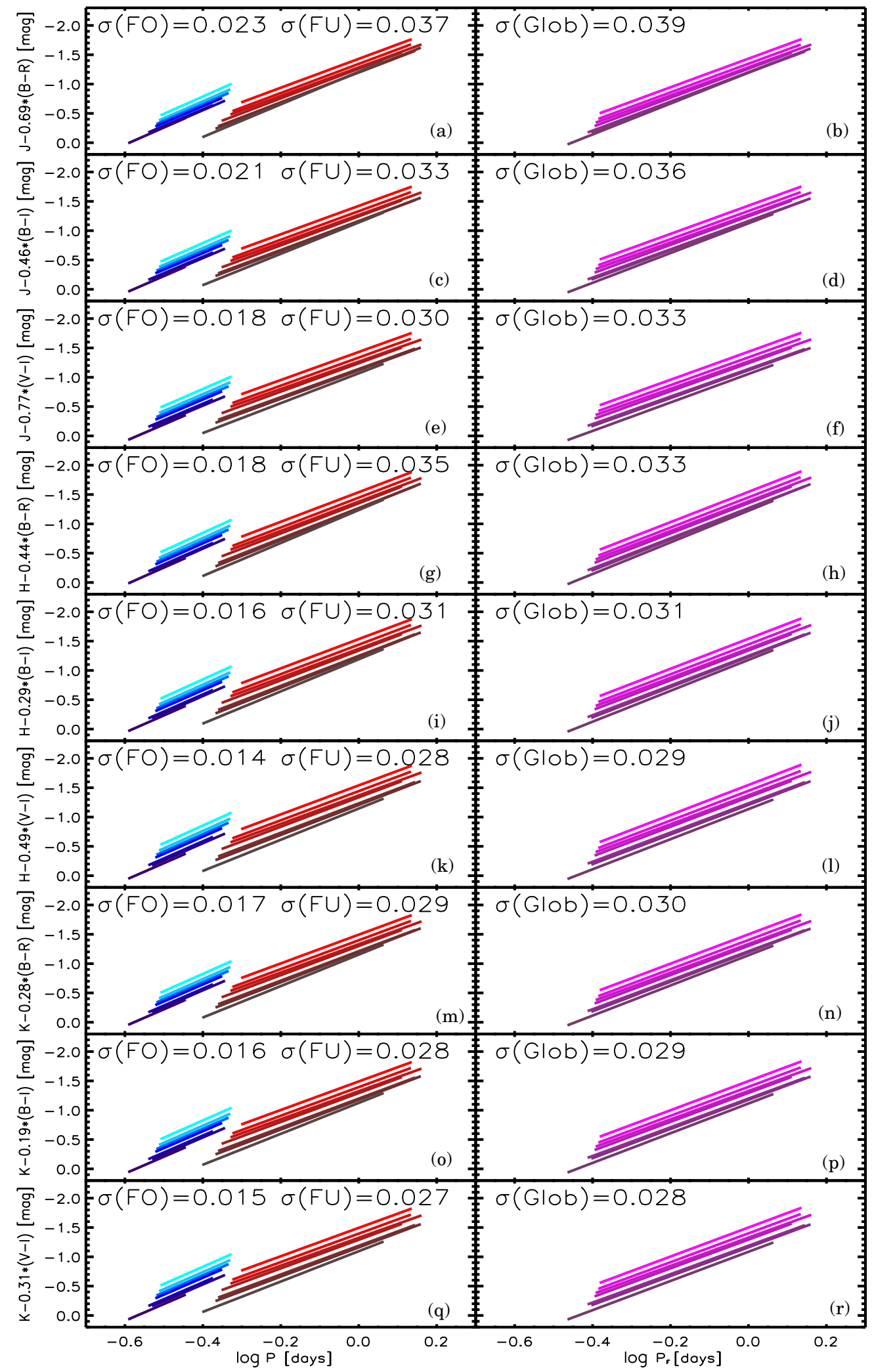

Figure 8. Same as Figure 6, but for the predicted optical-NIR three-band PW relations.

\subsection{Cluster Distances based on Predicted $P L Z$ and PWZ Relations}

We estimated the true distance modulus of M4 adopting the predicted PLZ relations discussed in the Section 5 together with the mean reddening $(E(B-V)=0.37 \pm 0.01 \mathrm{mag})$, the reddening law provided by $\mathrm{H} 12$ and a mean metal abundance of $[\mathrm{Fe} / \mathrm{H}]=-1.10$. The latter is a mean value based on iron abundances provided by Marino et al. $(2008,[\mathrm{Fe} / \mathrm{H}]=-1.13)$, Carretta et al. (2009, $[\mathrm{Fe} / \mathrm{H}]=-1.18)$ and by (Malavolta et al. 2014) using both RGB stars $([\mathrm{Fe} / \mathrm{H}]=-1.07)$ and main sequence stars and $([\mathrm{Fe} / \mathrm{H}]=-1.16)$. Note that to provide 


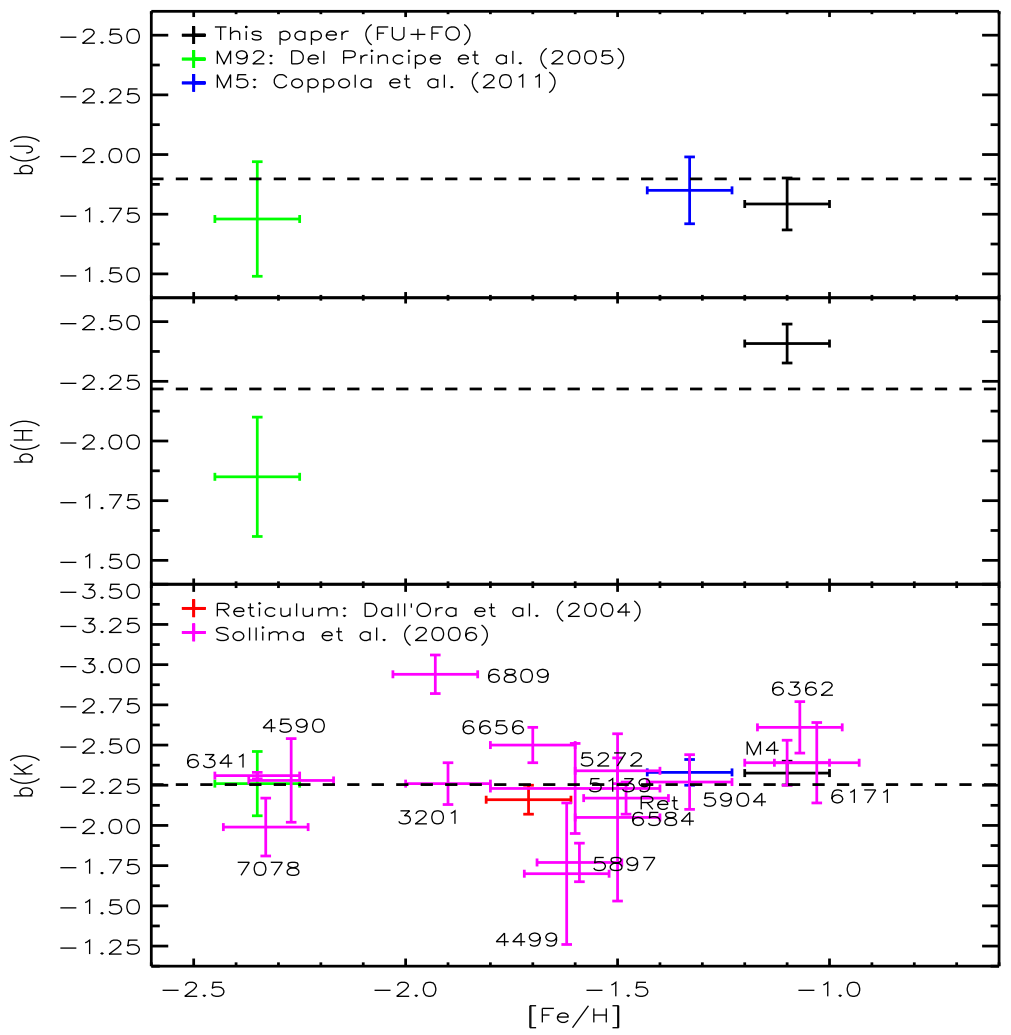

Figure 9. From top to bottom slopes of observed NIR PL relations as a function of the iron abundance. The iron abundances are based on the GC metallicity scale provided by Carretta et al. (2009). Top: slope of the $J$-band PL relation. The error bars display the error on the slope of the PL relations and the uncertainty on the metal abundance. The black line shows the slope of the predicted PLZ relation. Middle: same as the top, but for the $H$-band PL relation. Bottom: same as the top, but for the $K$-band PL relation.

homogeneous iron abundances the above measurements were rescaled to the same solar iron abundance adopted by Carretta et al. (2009). Moreover, to estimate individual distances we are using the mean apparent magnitudes together with the zeropoint and the slope of the predicted PLZ relations.

The results for true distance moduli are listed in Table 8 and plotted in Figure 10. The error on the distance modulus takes account of the photometric error, for uncertainties in the mean reddening $(E(B-V)=0.37 \pm 0.10 \mathrm{mag}))$, in the totalto-selective extinction ratio $\left(R_{V}=3.62 \pm 0.07\right)$, in the mean metallicity $(\sigma([\mathrm{Fe} / \mathrm{H}])=0.1 \mathrm{dex})$ and for the standard deviation of the adopted PLZ relation. The weighted true distance moduli based on FU, FO, and on the entire sample of variables agree within $1 \sigma$. However, the distance modulus based on FOs attains a smaller value compared with the FUs and with the combined sample (see labeled values). The main culprits are distance determinations based on optical PLZ relations, and indeed if we only use the NIR PLZ relations we find $\mu=11.266 \pm 0.014$ (error on the mean) \pm 0.025 (weighted standard deviation) mag for FOs, $11.271 \pm 0.012 \pm 0.020 \mathrm{mag}$ for FUs, and $11.283 \pm$ $0.010 \pm 0.018 \mathrm{mag}$ for the entire sample.

Note that the weighted standard deviation of the true distance moduli based on the $H$-band FO PLZ relations is smaller than in the $J$ and $K$ bands. The difference is due to the fact that the observed standard deviation in the $H$ band is smaller when compared with the $J$ and $K$ bands.

The true distance moduli based on optical, optical-NIR, and NIR PWZ relations show a more complex trend. The optical PWZ relations with vanishing metallicity terms display a large scatter when compared with true distance moduli based on the other NIR PWZ relations. In passing we note that the scatter

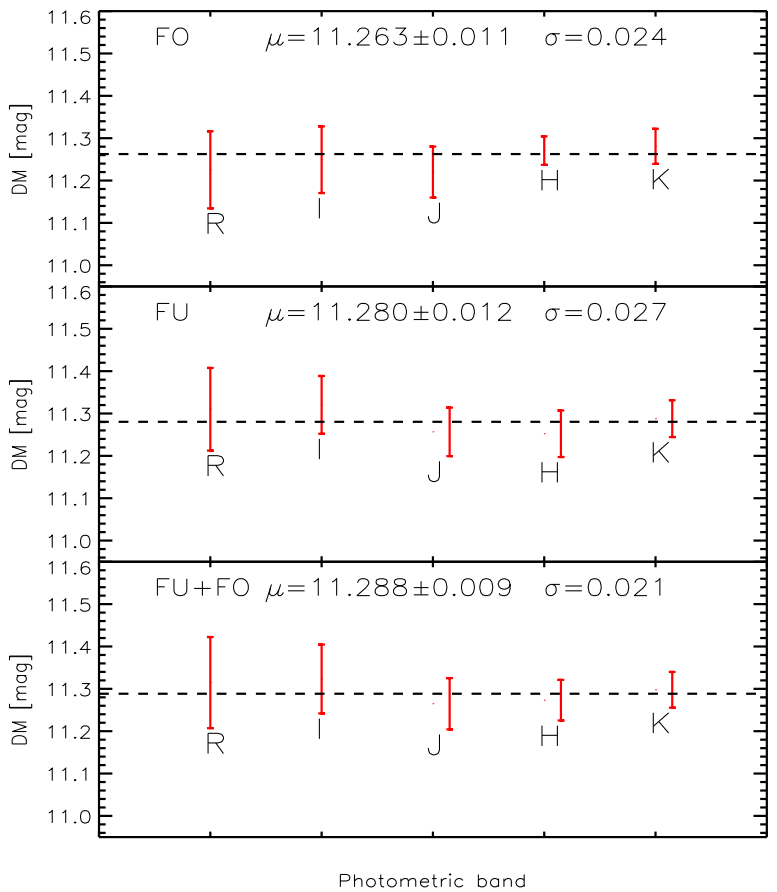

Figure 10. Top: true DM based on optical and NIR predicted FO PL relations that take account of the metallicity dependence. Following Carretta et al. (2009) we adopted for $\mathrm{M} 4$ an iron abundance of $[\mathrm{Fe} / \mathrm{H}]=-1.10$ The error bars include the photometric error, the extinction error, and the standard deviation of the adopted PL relation. The dashed lines shows the weighted mean true DM $(\mu)$. The error on the mean and the standard deviations are also labeled. Middle: same as the top, but for optical and NIR predicted FU PL relations. Bottom: same as the top, but for optical and NIR predicted PL relations including FU and FO RR Lyrae models. 
Table 8

True Distance Moduli based on Predicted Period-Luminosity-Metallicity Relations

\begin{tabular}{|c|c|c|c|c|c|c|}
\hline $\mathrm{PLZ}^{\mathrm{a}}$ & $\mu^{\mathrm{b}} \quad(\mathrm{mag})$ & $\sigma_{\mu}{ }^{\mathrm{c}}$ & $\mu^{\mathrm{b}} \quad(\mathrm{mag})$ & $\sigma_{\mu}^{\mathrm{c}}$ & $\mu^{\mathrm{b}} \quad(\mathrm{mag})$ & $\sigma_{\mu}^{\mathrm{c}}$ \\
\hline & FO & & FU & & $\mathrm{FU}+\mathrm{FO}$ & \\
\hline$R$ & $11.225 \pm 0.026$ & 0.091 & $11.310 \pm 0.018$ & 0.097 & $11.315 \pm 0.017$ & 0.108 \\
\hline$I$ & $11.249 \pm 0.023$ & 0.079 & $11.320 \pm 0.013$ & 0.068 & $11.323 \pm 0.013$ & 0.081 \\
\hline$J$ & $11.220 \pm 0.017$ & 0.060 & $11.257 \pm 0.010$ & 0.057 & $11.265 \pm 0.009$ & 0.061 \\
\hline$H$ & $11.271 \pm 0.009$ & 0.034 & $11.252 \pm 0.011$ & 0.055 & $11.273 \pm 0.008$ & 0.048 \\
\hline$K$ & $11.281 \pm 0.011$ & 0.041 & $11.288 \pm 0.008$ & 0.043 & $11.298 \pm 0.006$ & 0.042 \\
\hline
\end{tabular}

Notes.

a Adopted PLZ relation.

${ }^{\mathrm{b}}$ Mean distance modulus and its error based on FO, FU, and FU+FO variables.

c Standard deviation of the distance modulus based on FO, FU, and FU+FO variables.

Table 9

True Distance Moduli Based on Predicted Optical and NIR Period-Wesenheit-Metallicity Relations

\begin{tabular}{|c|c|c|c|c|c|c|}
\hline $\mathrm{PWZ}^{\mathrm{a}}$ & \multicolumn{2}{|c|}{ (mag) } & $\mu^{\mathrm{b}}$ & $\sigma_{\mu}^{\mathrm{c}}$ & \multicolumn{2}{|c|}{ (mag) } \\
\hline & \multicolumn{2}{|c|}{ FO } & \multicolumn{2}{|c|}{$\mathrm{FU}$} & \multicolumn{2}{|c|}{$\mathrm{FU}+\mathrm{FO}$} \\
\hline$V, B-V$ & $11.472 \pm 0.014$ & 0.050 & $11.372 \pm 0.019$ & 0.104 & $11.415 \pm 0.014$ & 0.093 \\
\hline$R, B-R$ & $11.338 \pm 0.017$ & 0.058 & $11.248 \pm 0.015$ & 0.077 & $11.287 \pm 0.013$ & 0.078 \\
\hline$R, V-R$ & $11.201 \pm 0.021$ & 0.073 & $11.103 \pm 0.014$ & 0.070 & $11.144 \pm 0.013$ & 0.082 \\
\hline$I, B-I$ & $11.326 \pm 0.014$ & 0.048 & $11.312 \pm 0.015$ & 0.080 & $11.329 \pm 0.011$ & 0.072 \\
\hline$I, R-I$ & $11.314 \pm 0.033$ & 0.116 & $11.335 \pm 0.016$ & 0.081 & $11.343 \pm 0.015$ & 0.091 \\
\hline$J, B-J$ & $11.232 \pm 0.013$ & 0.048 & $11.234 \pm 0.011$ & 0.063 & $11.247 \pm 0.009$ & 0.060 \\
\hline$J, V-J$ & $11.209 \pm 0.014$ & 0.051 & $11.223 \pm 0.012$ & 0.067 & $11.232 \pm 0.010$ & 0.063 \\
\hline$J, R-J$ & $11.209 \pm 0.015$ & 0.053 & $11.238 \pm 0.013$ & 0.072 & $11.242 \pm 0.011$ & 0.068 \\
\hline$J, I-J$ & $11.170 \pm 0.014$ & 0.048 & $11.238 \pm 0.018$ & 0.093 & $11.227 \pm 0.014$ & 0.087 \\
\hline$H, B-H$ & $11.270 \pm 0.004$ & 0.013 & $11.250 \pm 0.009$ & 0.044 & $11.274 \pm 0.007$ & 0.039 \\
\hline$H, V-H$ & $11.259 \pm 0.004$ & 0.013 & $11.246 \pm 0.010$ & 0.048 & $11.268 \pm 0.007$ & 0.041 \\
\hline$H, I-H$ & $11.247 \pm 0.005$ & 0.017 & $11.240 \pm 0.011$ & 0.053 & $11.260 \pm 0.008$ & 0.045 \\
\hline$K, B-K$ & $11.283 \pm 0.009$ & 0.029 & $11.272 \pm 0.008$ & 0.044 & $11.284 \pm 0.007$ & 0.044 \\
\hline$K, V-K$ & $11.277 \pm 0.009$ & 0.029 & $11.269 \pm 0.008$ & 0.045 & $11.280 \pm 0.007$ & 0.045 \\
\hline$K, R-K$ & $11.280 \pm 0.009$ & 0.029 & $11.278 \pm 0.008$ & 0.045 & $11.288 \pm 0.007$ & 0.044 \\
\hline$K, I-K$ & $11.277 \pm 0.010$ & 0.030 & $11.271 \pm 0.009$ & 0.048 & $11.281 \pm 0.007$ & 0.047 \\
\hline$K, J-K$ & $11.323 \pm 0.008$ & 0.029 & $11.293 \pm 0.012$ & 0.060 & $11.313 \pm 0.009$ & 0.058 \\
\hline \multicolumn{7}{|c|}{ Three-bands } \\
\hline$J, B-R$ & $11.258 \pm 0.013$ & 0.044 & $11.233 \pm 0.011$ & 0.061 & $11.255 \pm 0.009$ & 0.058 \\
\hline$J, B-I$ & $11.250 \pm 0.011$ & 0.036 & $11.251 \pm 0.008$ & 0.042 & $11.264 \pm 0.007$ & 0.041 \\
\hline$J, V-I$ & $11.226 \pm 0.012$ & 0.040 & $11.235 \pm 0.009$ & 0.047 & $11.246 \pm 0.007$ & 0.046 \\
\hline$H, B-R$ & $11.277 \pm 0.004$ & 0.015 & $11.240 \pm 0.008$ & 0.038 & $11.270 \pm 0.007$ & 0.040 \\
\hline$H, B-I$ & $11.284 \pm 0.003$ & 0.011 & $11.255 \pm 0.009$ & 0.042 & $11.282 \pm 0.006$ & 0.038 \\
\hline$H, V-I$ & $11.272 \pm 0.003$ & 0.009 & $11.252 \pm 0.010$ & 0.048 & $11.275 \pm 0.007$ & 0.040 \\
\hline$K, B-R$ & $11.289 \pm 0.009$ & 0.028 & $11.270 \pm 0.009$ & 0.049 & $11.284 \pm 0.008$ & 0.047 \\
\hline
\end{tabular}

Notes.

a Adopted PWZ relation.

${ }^{\mathrm{b}}$ Mean distance modulus and its error based on FO, FU, and FU+FO variables.

${ }^{\mathrm{c}}$ Standard deviation of the distance modulus based on FO, FU, and FU+FO variables.

of the true distance moduli based on the optical PWZ relations slightly decreases when using PW relations that neglect the metallicity dependence.

Among the true distance moduli based on optical-NIR PWZ relations those including the $J$ band attain slightly smaller values (see Table 9 and Figure 11). The reason for the difference it is not clear. The adopted color-temperature relations to transform the theoretical models into the observational plane might be a possible culprit. A similar difference was also found in optical and in
optical-NIR color-magnitude diagrams by Bono et al. (2010b). The weighted standard deviations of the true distance moduli of FO PWZ relations including the $H$ band are smaller than in the $J$ and $K$ bands. The reasons for the difference are the same as for the PLZ relation. Interestingly enough, the true distance moduli based on optical-NIR PWZ relations including the $H$ and $K$ bands are very accurate and display a very small dispersion.

The weighted mean true distance modulus based on optical, optical-NIR, and NIR PWZ relations agrees within $1 \sigma$. 


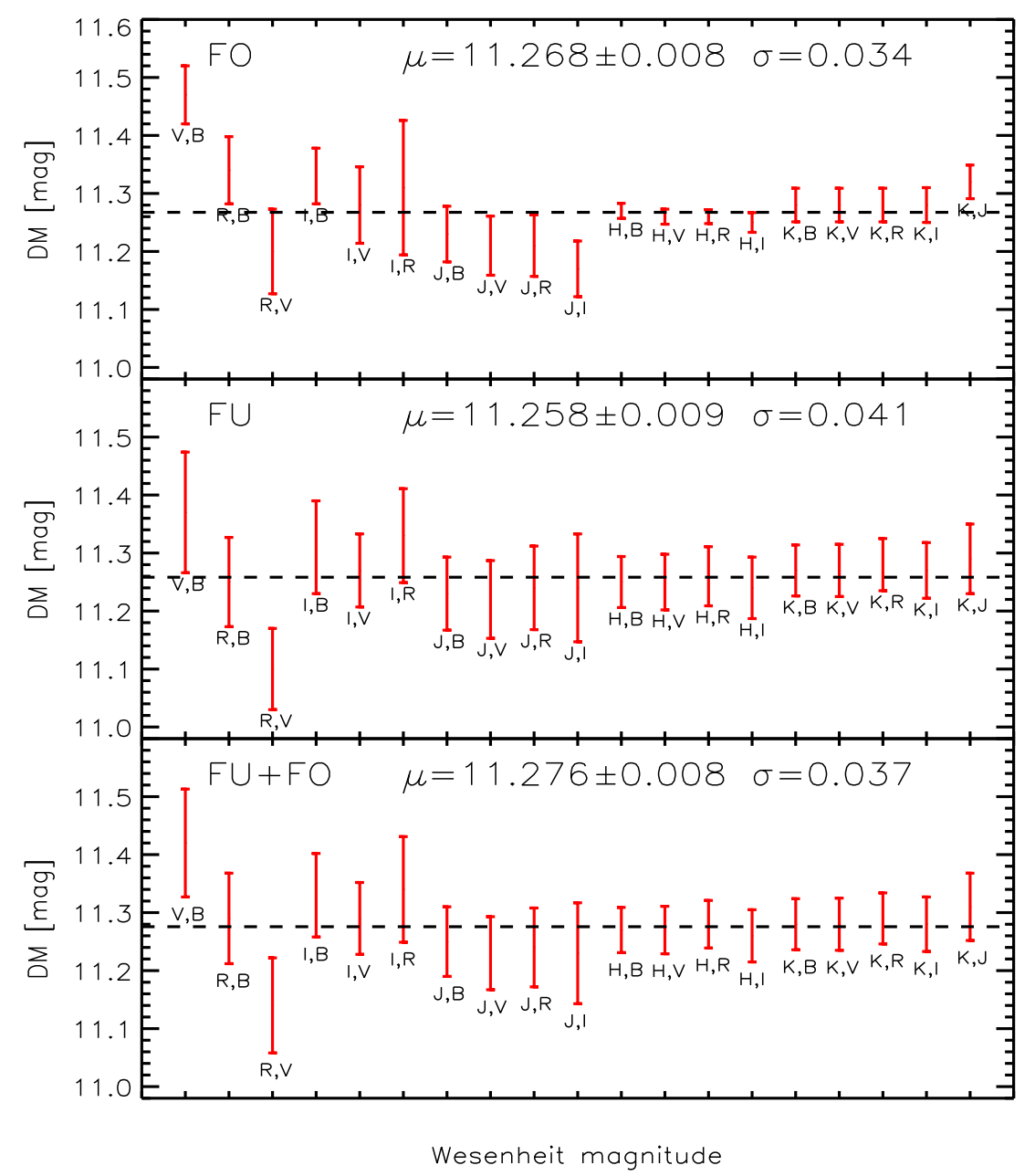

Figure 11. Top: true DM based on optical and NIR predicted FO PW relations that take account of the metallicity dependence. Following Carretta et al. (2009) we adopted for M4 an iron abundance of $[\mathrm{Fe} / \mathrm{H}]=-1.10$ The error bars include the photometric error, the extinction error, and the standard deviation of the adopted PW relation. The dashed lines shows the weighted mean true DM $(\mu)$. The error on the mean and the standard deviations are also labeled. Middle: same as the top, but for optical and NIR predicted FU PW relations. Bottom: same as the top, but for optical and NIR predicted PW relations including FU and FO RR Lyrae models.

The agreement between the three different sets of distance determinations minimally improves if we only use optical-NIR and NIR PWZ relations: $11.263 \pm 0.006 \pm 0.021 \mathrm{mag}$ for FOs, $11.259 \pm 0.005 \pm 0.019 \mathrm{mag}$ for FUs, and $11.272 \pm 0.005 \pm$ $0.019 \mathrm{mag}$ for the entire sample. The lack of a clear dependence of the estimated distance moduli on the photometric bands is further supporting the accuracy of the reddening law adopted to estimate the PW relations and the true mean optical and NIR magnitudes (Pietrzynski et al. 2006).

Distance moduli based on three-band PWZ relations have, as expected, a smaller dispersion when compared with twoband ones (see Figure 12). In particular, the weighted mean true distance modulus based on FOs is $11.275 \pm 0.004 \pm$ $0.011 \mathrm{mag}$ for FOs, while those based on FUs is $11.254 \pm$ $0.005 \pm 0.014 \mathrm{mag}$ for FUs, and those based on the entire sample is $11.272 \pm 0.004 \pm 0.013 \mathrm{mag}$.

The current distance evaluations based on predicted PLZ and PWZ relations agree with each other within $1 \sigma$. They also agree quite well with distance determinations to M4 based on solid standard candles, and in particular, with the distance recently provided by Kaluzny et al. (2013), who obtained a value of $11.30 \pm 0.05 \mathrm{mag}$ using three eclipsing binaries. The same outcome applies to the recent distance evaluation based on the HB luminosity level (11.28 $\pm 0.06 \mathrm{mag})$ provided by H12. The above findings indicate that NIR PLZ relations and optical-NIR/NIR PWZ relations can provide individual distances to GCs hosting a good sample of RRLs with a precision better than $2 \%-3 \%$.

\section{FINAL REMARKS AND CONCLUSIONS}

We have presented new and precise optical, optical-NIR, and NIR PL relations and PW relations. We have provided independent empirical relations for $\mathrm{FO}, \mathrm{FU}$, and for the entire sample of RRLs in M4.

The mean weighted visual apparent magnitude of 44 cluster RRLs is $\langle V\rangle=13.329 \pm 0.000 \pm 0.177 \mathrm{mag}$, where the former error refers to the error on the mean and the latter to the weighted standard deviation. The current estimate agrees quite well with similar evaluations available in the literature. Indeed, Liu \& Janes (1990b) using four FU variables (V2, V15, V32, V33) found $\langle V\rangle=13.287 \pm 0.025 \pm 0.213 \mathrm{mag}$, while Clementini et al. (1994) using four FU variables (V2, V15, V29, V42) found $\langle V\rangle=13.371 \pm 0.001 \pm 0.139 \mathrm{mag}$. This is a relevant stepping stone for the forthcoming investigation in which we plan to estimate the absolute (Bono et al. 2010b) and the relative (VandenBerg et al. 2013) age of M4 using both optical and NIR photometry.

We have estimated the true distance modulus to M4 using the observed slopes and RR Lyr itself to fix the zero-point. RR Lyr is, 


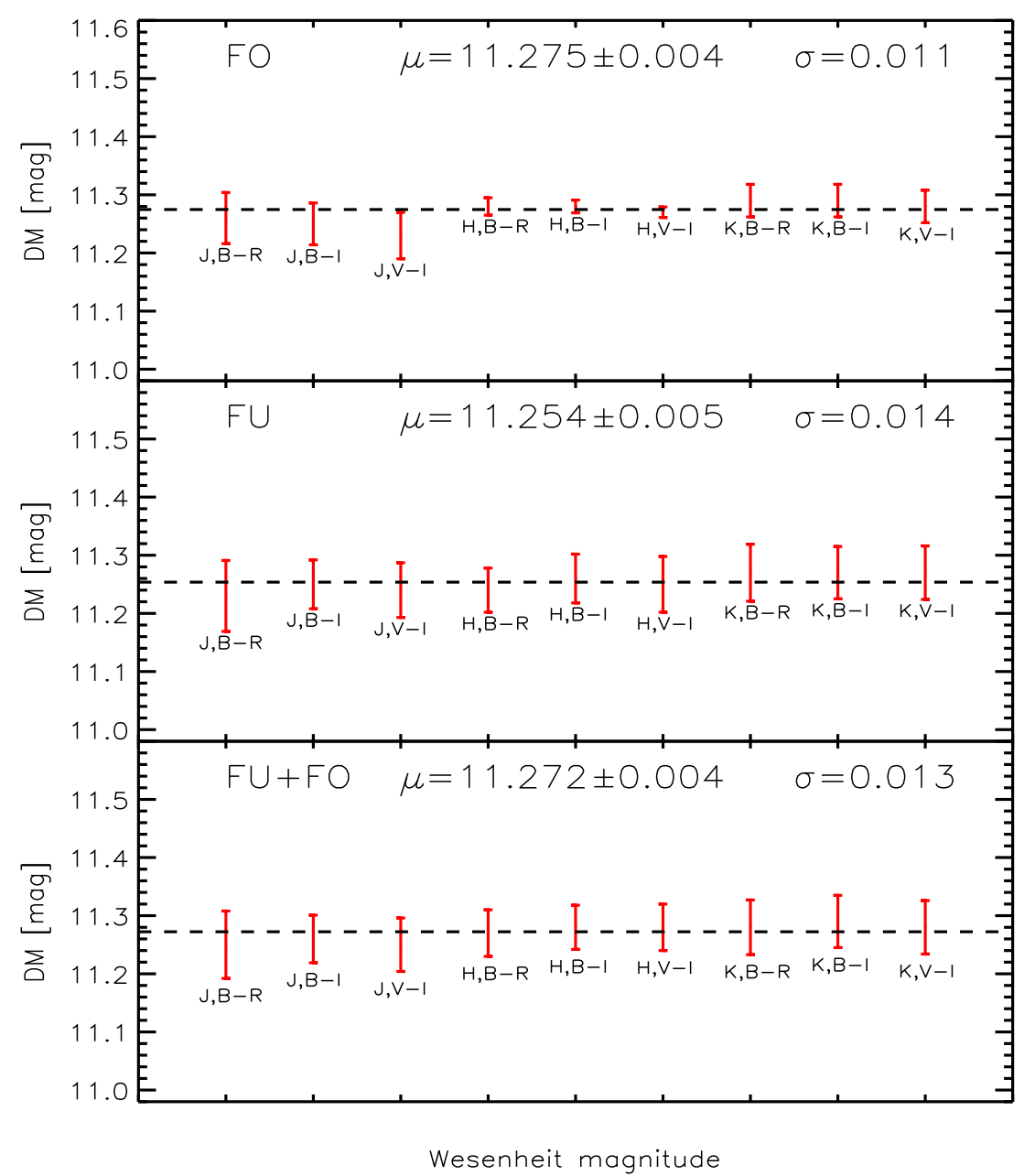

Figure 12. Same as Figure 11, but the true distance moduli were estimated by adopting optical-NIR, three-band PWZ relations. The optical color adopted in the PWZ relation is independent of the adopted NIR magnitude.

out of the five field RRLs with accurate trigonometric parallaxes measured by HST (Benedict et al. 2011), the calibrator with the most precise distance and with both optical $(B V)$ and NIR $(J H K)$ mean magnitudes. Moreover, accurate estimates of both the iron content and the reddening are also available. The main drawback in using RR Lyr is that it is affected by the Blazhko effect together with UV Oct and XZ Cyg. The impact is minimal in the NIR bands, but the uncertainty in the mean optical bands is of the order of $0.10 \mathrm{mag}$. To determine the true distance modulus we took account of the difference in iron abundance between RR Lyr and M4 $(\Delta[\mathrm{Fe} / \mathrm{H}] \sim 0.40$, according to the metallicity scale by Carretta et al. 2009). The difference was estimated using predicted PLZ and PWZ relations. The weighted mean true distance modulus based on three independent empirical NIR PL relations and the entire sample of RRLs (FO+FU) is $11.35 \pm 0.03 \pm 0.05 \mathrm{mag}$. The weighted mean true distance modulus based on eight different empirical optical-NIR and NIR PW relations is $11.32 \pm 0.02 \pm 0.07 \mathrm{mag}(\mathrm{FO}+\mathrm{FU})$. The above estimates agree quite well with similar estimates available in the literature and within $1 \sigma$ with distances based on predicted PLZ and PWZ relations.

We also estimated the true distance moduli to M4 using predicted optical, optical-NIR, and NIR PLZ and PWZ relations. The theoretical relations are based on a broad range of nonlinear, convective pulsation models for RRLs. They were constructed for both FO and FU pulsators and cover a broad range in stellar masses $\left(M=0.80-0.55 M_{\odot}\right)$ and metal abundances $(Z=0.0001-0.02)$.

The true distance moduli based on the PLZ relations take account of uncertainties in the mean reddening, in the photometry, in the mean metallicity and in the standard deviation of the adopted PLZ relation. The true distance moduli based on the PWZ relations take account of uncertainties in the photometry and in the mean metallicity.

We found that true distance moduli based on NIR PLZ relations are, as expected, characterized by smaller intrinsic dispersions when compared with optical PLZ relations. The difference is mainly the consequence of steeper slopes in the PLZ relation at longer wavelengths.

We also found that optical PWZ relations present larger intrinsic dispersions when compared with optical-NIR and NIR PWZ relations. The difference is mainly the consequence of a nonlinear dependence on the metallicity in the optical regime when compared with the optical-NIR and with the NIR regimes.

True distance moduli based on FO PLZ and PWZ relations display smaller weighted standard deviations when compared with FU, and FO+FU PLZ and PWZ relations. This evidence-taken at face value - seems to argue in favor of the idea that FO variables can provide accurate and precise individual distance moduli. However M4 hosts a dozen of FO RRLs, further support based on GCs hosting sizable samples of FO pulsators is required. 
Recent findings based on MIR photometry collected with WISE of field RRLs strongly suggest a very small intrinsic scatter in the PL relation and a mild dependence on the metal abundance (Madore et al. 2013; Klein et al. 2014). The use of MIR photometry collected with Spitzer within the Carnegie RR Lyrae Project appears as a natural development of this investigation. The key advantage in this approach is that we can use the five empirical calibrators for which are available accurate MIR mean magnitudes.

The above findings appear very promising not only for the next generation of extremely large telescopes (ELTs), namely the European-ELT, ${ }^{9}$ the Thirty Meter Telescope, ${ }^{10}$ and the Giant Magellan Telescope, ${ }^{11}$ but also for James Web Space Telescope ${ }^{12}$ and EUCLID. ${ }^{13}$ Future ground-based and space facilities will be equipped with a suite of NIR and MIR detectors to perform accurate photometry and spectroscopy of old stellar tracers in the nearby universe. This is an unique opportunity to improve the cosmic distance scale of stellar systems hosting old stellar populations (early and late type galaxies) from the Local Group to the Virgo galaxy cluster.

It is a pleasure to thank the organizers of the MIAPP-Munich Institute for Astro and Particle Physics-workshop on The Extragalactic Distance Scale. During the workshop several authors have had the opportunity to present the preliminary results of this investigation and to discuss pros and cons of the RR Lyrae distance scale in a very fruitful and pleasant environment. In addition we warmly thank R.P. Kudritzski for many useful discussions and insights concerning stellar atmospheres and non-LTE effects in giant stars. We also would like to thank an anonymous referee for several suggestions concerning the content and the cut of an early version of the current manuscript. This work was partially supported by PRIN-INAF 2011 "Tracing the formation and evolution of the Galactic halo with VST" (P.I.: M. Marconi) and by PRIN-MIUR (2010LY5N2T) "Chemical and dynamical evolution of the Milky Way and Local Group galaxies" (P.I.: F. Matteucci). One of us (G.B.) thanks The Carnegie Observatories visitor programme for support as science visitor. This publication makes use of data products from the Two Micron All Sky Survey, which is a joint project of the University of Massachusetts and the Infrared Processing and Analysis Center/California Institute of Technology, funded by the National Aeronautics and Space Administration and the National Science Foundation.

\section{REFERENCES}

Andrievsky, S. M., Kovtyukh, V. V., Wallerstein, G., Korotin, S. A., \& Huang, W. 2010, PASP, 122, 877

Asplund, M., Grevesse, N., \& Sauval, A. J. 2005, in ASP Conf. Ser. 336, Cosmic Abundances as Records of Stellar Evolution and Nucleosynthesis in Honor of David L. Lambert, ed. T. G. Barnes, III \& F. N. Bash (San Francisco, CA: ASP), 25

Bedin, L. R., Salaris, M., Piotto, G., et al. 2009, ApJ, 697, 965

Benedict, G. F., McArthur, B. E., Feast, M. W., et al. 2011, AJ, 142, 187

Benedict, G. F., McArthur, B. E., Fredrick, L. W., et al. 2002, AJ, 123, 473

Benkho, J. M., Bakos, G.Á., \& Nuspl, J. 2006, MNRAS, 372, 1657

Bond, H. E., Nelan, E. P., VandenBerg, D. A., Schaefer, G. H., \& Harmer, D. 2013, ApJ, 765, 12

Bono, G. 2003, LNP, 635, 85

\footnotetext{
9 http://www.eso.org/public/teles-instr/e-elt.html

10 http://www.tmt.org/

11 http://www.gmto.org/

12 http://www.jwst.nasa.gov/

13 http://sci.esa.int/euclid/
}

Bono, G., Caputo, F., Castellani, V., Marconi, M., \& Storm, J. 2001, MNRAS, 326, 1183

Bono, G., Caputo, F., Castellani, V., Marconi, M., \& Storm, J. 2002, MNRAS, 332,78

Bono, G., Caputo, F., Castellani, V., et al. 2003, MNRAS, 344, 1097

Bono, G., Caputo, F., Fiorentino, G., Marconi, M., \& Musella, I. 2008a, ApJ, 684, 102

Bono, G., Caputo, F., Marconi, M., \& Musella, I. 2010a, ApJ, 715, 277

Bono, G., Caputo, F., \& Stellingwerf, R. F. 1994, ApJ, 432, 51

Bono, G., Dall'Ora, M., Caputo, F., et al. 2011, in Carnegie Obs. Astrophys. Ser. 5, RR Lyrae Stars, Metal-Poor Stars, and the Galaxy, ed. A. McWilliam (Pasadena, CA: Carnegie Observatories), 1

Bono, G., \& Stellingwerf, R. F. 1994, ApJS, 93, 233

Bono, G., Stetson, P. B., Sanna, N., et al. 2008b, ApJL, 686, L87

Bono, G., Stetson, P. B., VandenBerg, D. A., et al. 2010b, ApJL, 708, L74

Buonanno, R., Corsi, C. E., \& Fusi Pecci, F. 1989, A\&A, 216, 80

Buonanno, R., Corsi, C. E., Pulone, L., Fusi Pecci, F., \& Bellazzini, M. 1998, A\&A, 333, 505

Caputo, F., Castellani, V., Marconi, M., \& Ripepi, V. 2000, MNRAS, 316, 819

Capuzzo-Dolcetta, R., \& Miocchi, P. 2008, ApJ, 681, 1136

Cardelli, J. A., Clayton, G. C., \& Mathis, J. S. 1989, ApJ, 345, 245

Carney, B. W., Storm, J., \& Jones, R. V. 1992, ApJ, 386, 663

Carretta, E., Bragaglia, A., Gratton, R., D’Orazi, V., \& Lucatello, S. 2009, A\&A, 508,695

Casetti-Dinescu, D. I., Girard, T.M, Herrera, D., et al. 2007, AJ, 134, 195

Casetti-Dinescu, D. I., Girard, T.M, Jílková, L., et al. 2013, AJ, 146, 33

Cassisi, S., Castellani, M., Caputo, F., \& Castellani, V. 2004, A\&A, 426, 641

Cassisi, S., Potekhin, A. Y., Pietrinferni, A., Catelan, M., \& Salaris, M. 2007, ApJ, 661, 1094

Castelli, F., Gratton, R. G., \& Kurucz, R. L. 1997a, A\&A, 318, 841

Castelli, F., Gratton, R. G., \& Kurucz, R. L. 1997b, A\&A, 324, 432

Catelan, M., Pritzl, B. J., \& Smith, H. A. 2004, ApJS, 154, 633

Clementini, G., Merighi, R., Pasquini, L., Cacciari, C., \& Gouiffes, C. 1994, MNRAS, 267, 83

Cohen, R. E., \& Sarajedini, A. 2012, MNRAS, 419, 342

Coppola, G., Dall'Ora, M., Ripepi, V., et al. 2011, MNRAS, 416, 1056

Cox, A. N., Hodson, S. W., \& Clancy, S. P. 1983, ApJ, 266, 94

Dall'Ora, M., Storm, J., Bono, G., et al. 2004, ApJ, 610, 269

De Angeli, F., Piotto, G., Cassisi,, et al. 2005, AJ, 130, 116

Del Principe, M., Piersimoni, A. M., Bono, G., et al. 2005, AJ, 129, 2714

Del Principe, M., Piersimoni, A. M., Storm, J., et al. 2006, ApJ, 652, 362

Denissenkov, P. A., \& VandenBerg, D. A. 2003, ApJ, 593, 509

Di Cecco, A., Zocchi, A., Varri, A. L., et al. 2013, AJ, 145, 103

Di Criscienzo, M., Greco, C., Ripepi, V., et al. 2011, AJ, 141, 81

Dixon, R. I., \& Longmore, A. J. 1993, MNRAS, 265, 395

Dotter, A., Chaboyer, B., Javremović, D., et al. 2007, AJ, 134, 376

Dotter, A., Sarajedini, A., \& Anderson, J. 2011, ApJ, 738, 74

Feast, M., Whitelock, P., \& Menzies, J. 2002, MNRAS, 329, 7

Ferraro, F. R., Messineo, M., Fusi Pecci, F., et al. 1999, AJ, 118, 1738

Fiorentino, G., Musella, I., \& Marconi, M. 2013, MNRAS, 434, 2866

Gilliland, R. L., Bono, G., Edmonds, P. D., et al. 1998, ApJ, 507, 818

Gratton, R. G., Bragaglia, A., Carretta, E., et al. 2003, A\&A, 408, 529

Gratton, R. G., Sneden, C., \& Carretta, E. 2004, ARA\&A, 42, 385

Hansen, B. M. S., Richer, H. B., Fahlman, G. G., et al. 2004, ApJS, 155, 551

Harris, W. E. 1996, AJ, 112, 1487

Hendricks, B., Stetson, P. B., VandenBerg, D. A., \& Dall'Ora, M. 2012, AJ, 144,25

Iben, I., Jr., \& Huchra, J. 1971, A\&A, 14, 293

Inno, L., Matsunaga, N., Bono, G., et al. 2013, ApJ, 764, 84

Jones, R. V., Carney, B. W., \& Fulbright, J. P. 1996, PASP, 108, 877

Jones, R. V., Carney, B. W., \& Latham, D. W. 1988, ApJ, 332, 206

Johnson, C. I., Rich, R. M., Kobayashi, C., et al. 2013, ApJ, 765, 157

Kaluzny, J., \& Thompson, I. B. 2009, AcA, 59, 273

Kaluzny, J., Thompson, I. B., Rozyczka, M., Dotter, A., \& Krzeminski, W. 2013, AJ, 145, 43

Klein, C. R., Richards, J. W., Butler, N. R., \& Bloom, J. S. 2014, MNRAS, 440, 96L

Kolenberg, K., Fossati, L., Shulyak, D., et al. 2010, A\&A, 519, 64

Kolenberg, K., Smith, H. A., Gazeas, K. D., et al. 2006, A\&A, 459, 577

Kouwenhoven, M. B. N., Goodwin, S. P., Parker, R. J., et al. 2010, MNRAS, 404, 1835

Kraft, R. P., \& Ivans, I. I. 2003, PASP, 115, 143

Kudritzki, R. P. 1979, LIACo, 22, 295

Lardo, C., Pancino, E., Mucciarelli, A., et al. 2013, MNRAS, 433, 1941

Layden, A. C., Sarajedini, A., von Hippel, T., \& Cool, A. M. 2005, ApJ, 632,266

Leaman, R., VandenBerg, D. A., \& Mendel, J. T. 2013, MNRAS, 436, 122 
Liu, T., \& Janes, K. A. 1990a, ApJ, 354, 273

Liu, T., \& Janes, K. A. 1990b, ApJ, 360, 561

Longmore, A. J., Dixon, R., Skillen, I., Jameson, R. F., \& Fernley, J. A. 1990, MNRAS, 247, 684

Longmore, A. J., Fernley, J. A., \& Jameson, R. F. 1986, MNRAS, 220, 279

Madore, B. F. 1982, ApJ, 253, 575

Madore, B. F., Hoffman, D., Freedman, W. L., et al. 2013, ApJ, 776, 135

Majaess, D., Turner, D., \& Gieren, W. 2012a, PASP, 124, 1035

Majaess, D., Turner, D., Gieren, W., \& Lane, D. 2012b, ApJ, 752, 10

Malavolta, L., Sneden, C., Piotto, G., et al. 2014, AJ, 147, 25

Marconi, M., Bono, G., Caputo, F., et al. 2011, ApJ, 738, 111

Marconi, M., Nordgren, T., Bono, G., Schnider, G., \& Caputo, F. 2005, ApJL, 623, L133

Marín-Franch, A., Aparicio, A., Piotto, G., et al. 2009, ApJ, 694, 1498

Marino, A. F., Villanova, S., Piotto, G., et al. 2008, A\&A, 490, 625

Matsunaga, N., Feast, M. W., \& Menzies, J. W. 2009, MNRAS, 397, 933

McNamara, D. H. 2011, AJ, 142, 110

Milone, A. P., Marino, A. F., Piotto, G., et al. 2013, ApJ, 767, 120

Nardetto, N., Fokin, A., Fouqué, P., et al. 2013, A\&A, 534, 16

Perryman, M. A. C., Lindegren, L., Kovalevsky, J., et al. 1997, A\&A, 323, 49

Peterson, R. C., Carney, B. W., \& Latham, D. W. 1996, ApJ, 465, 47

Peterson, R. C., Rees, R. F., \& Cudworth, K. M. 1995, ApJ, 453, 214

Pietrinferni, A., Cassisi, S., Salaris, M., \& Castelli, F. 2004, ApJ, 612, 168

Pietrinferni, A., Cassisi, S., Salaris, M., \& Castelli, F. 2006, ApJ, 642, 797

Pietrinferni, A., Cassisi, S., Salaris, M., \& Hidalgo, S. 2013, A\&A, 558, 46

Pietrzyński, G., Gieren, W., Soszynski, I., et al. 2006, AJ, 132, 2556

Poleski, R., Udalski, A., Gould, A., et al. 2013, ApJ, 776, 76

Preston, G. W., \& Chadid, M. 2013, EAS, 63, 35

Pulone, L. 1992, MmSAI, 63, 485

Richer, H. B., Brewer, J., Fahlman, G. G., et al. 2004, AJ, 127, 2904

Richer, H. B., Fahlman, G. G., Ibata, R. A., et al. 1997, ApJ, 484, 741

Richer, H. B., Goldsbury, R., Heyl, J., et al. 2013, ApJ, 778, 104

Riess, A. G., Macri, L., Casertano, S., et al. 2011, ApJ, 730, 119
Roederer, I. U., Marino, A. F., \& Sneden, C. 2011, ApJ, 742, 37

Rood, R. T. 1973, AJ, 184, 815

Salaris, M. 2012, Ap\&SS, 341, 65

Salaris, M. 2013, in IAU Symp. 289, Advancing the Physics of Cosmic Distances, ed. R. de Grijs (Cambridge: Cambridge Univ. Press), 145

Salaris, M., Riello, M., Cassisi, S., \& Piotto, G. 2004, A\&A, 420, 911

Sarajedini, A., Bedin, L. R., Chaboyer, B., et al. 2007, AJ, 133, 1658

Skrutskie, M. F., Cutri, R. M., Stiening, R., et al. 2006, AJ, 131, 1163

Sollima, A., Cacciari, C., Arkharov, A. A. H., et al. 2008, MNRAS, 384, 1583

Sollima, A., Cacciari, C., \& Valenti, E. 2006, MNRAS, 372, 1675

Spite, M., Spite, F., \& Bonifacio, P. 2012, MSAIS, 22, 9

Stetson, P. B. 2000, PASP, 112, 925

Stetson, P. B. 2005, PASP, 117, 563

Stetson, P. B., Bolte, M., Harris, W. E., et al. 1999, AJ, 117, 247

Stetson, P. B., Braga, V. F., \& Dall'Ora, M. 2014, PASP, 126, 521

Szeidl, B., Guinan, E. F., Olah, K., \& Szabados, L. 1997, CoKon, 99, 1

Thompson, I. B., Kaluzny, J., Rucinski, S. M., et al. 2010, AJ, 139, 329

Troisi, F., Bono, G., Stetson, P. B., et al. 2011, PASP, 123, 879

Valenti, E., Origlia, L., \& Rich, R. M. 2011, MNRAS, 414, 2690

van Altena, W. F., Lee, J. T., \& Hoffleit, E. D. 1995, The General Catalogue of Trigonometric [Stellar] Parallaxes (4th ed.; New Haven, CT: Yale Univ. Observatory)

Van den Bergh, S. 1975, in Stars and Stellar Systems, 9, 509, ed. A. Sandage, M. Sandage, \& J. Kristian (Chicago: Univ. Chicago Press)

VandenBerg, D. A., Bergbusch, P. A., Dotter, A., et al. 2012, ApJ, 755, 15

VandenBerg, D. A., Brogaard, K., Leaman, R., \& Casagrande, L. 2013, ApJ, 775,134

Vieira, K., Casetti-Dinescu, D. I., Méndez, R. A., et al. 2007, AJ, 134, 1432

Zinn, R. 1980, ApJS, 42, 19

Zinn, R., \& West, M. J. 1984, ApJS, 55, 45

Zoccali, M., Cassisi, S., Frogel, J. A., et al. 2000, ApJ, 530, 418

Zoccali, M., Renzini, A., Ortolani, S., et al. 2001, ApJ, 553, 733

Zoccali, M., Renzini, A., Ortolani, S., et al. 2003, A\&A, 399, 931 